\title{
RMetS
}

Royal Meteorological Society

\section{The TKE budget in the convective Martian planetary boundary layer}

\author{
G. M. Martínez, ${ }^{\mathrm{a}, \mathrm{c} *}$ F. Valero ${ }^{\mathrm{b}}$ and L. Vázquez ${ }^{\mathrm{c}}$ \\ ${ }^{a}$ Department of Atmospheric, Oceanic, and Space Sciences, University of Michigan, Ann Arbor, USA \\ ${ }^{\mathrm{b}}$ Facultad de Física, Universidad Complutense de Madrid, Spain \\ ${ }^{\mathrm{c}}$ Facultad de Informática, Universidad Complutense de Madrid, Spain
}

${ }^{*}$ Correspondence to: G. M. Martínez, Department of Atmospheric, Oceanic, and Space Sciences, University of Michigan, 2455 Hayward St., 48109 Ann Arbor, USA. E-mail: gemartin@umich.edu

The turbulent kinetic energy (TKE) budget has been obtained for the first time from ground-based data on Mars, both for the unstable surface layer (SL) and for the convectively driven mixed layer (CML). Values for storage, buoyancy, shear, vertical turbulent transport, dissipation, and an imbalance term accounting for the rest of the TKE budget have been determined and weighted for significance.

These results have been derived from ground-based measurements made by Viking Lander 1 (VL1) on Sol 28, Viking Lander 2 (VL2) on Sol 20, and Pathfinder (PF) on Sol 25, and through an adaptation to Mars of terrestrial similarity theory, which constitutes a new approach to the study of the TKE budget on Mars.

Shear is the main TKE generator in the unstable SL for VL1 Sol 28 and PF Sol 25. It is narrowly exceeded by dissipation, the main mechanism removing TKE. Both terms present values $\sim 10^{-1} \mathrm{~m}^{2} \mathrm{~s}^{-3}$. Buoyancy generates TKE, though it plays a minor role $\left(\sim 10^{-2} \mathrm{~m}^{2} \mathrm{~s}^{-3}\right)$. Vertical turbulent transport balances buoyancy, removing TKE from the SL by sending it upwards. The imbalance term represents $30 \%$ of the main mechanisms, while storage plays an insignificant role $\left(\sim 10^{-5} \mathrm{~m}^{2} \mathrm{~s}^{-3}\right)$. The SL TKE budget for VL2 Sol 20 presents a different behaviour instead, with the imbalance term becoming the main TKE generator, likely due to the anomalous atmospheric conditions existing during this Sol.

Buoyancy and dissipation play the major roles generating and removing TKE in the CML for the three Sols under study, respectively, both showing values around $5 \times 10^{-3} \mathrm{~m}^{2} \mathrm{~s}^{-3}$. Vertical turbulent transport plays a minor role $\left(\sim 10^{-4} \mathrm{~m}^{2} \mathrm{~s}^{-3}\right)$, and so does the imbalance term, with values about $25 \%$ of buoyancy or dissipation. Finally, shear and storage terms are negligible, presenting values $\sim 10^{-6}$ and $\sim 10^{-5} \mathrm{~m}^{2} \mathrm{~s}^{-3}$, respectively. Copyright (c) 2011 Royal Meteorological Society

Key Words: Mars; turbulent kinetic energy; ground-based data; convective conditions; similarity theory

Received 24 November 2010; Revised 23 March 2011; Accepted 17 June 2011; Published online in Wiley Online Library 12 August 2011

Citation: Martínez GM, Valero F, Vázquez L. 2011. The TKE budget in the convective Martian planetary boundary layer. Q. J. R. Meteorol. Soc. 137: 2194-2208. DOI:10.1002/qj.883

\section{Introduction}

The turbulent kinetic energy (TKE) is a measure of the intensity of the turbulence, and one of the most important variables in micrometeorology (Arya, 2001). Advection, buoyancy, shear, turbulent transport, turbulent pressure redistribution, and dissipation are the mechanisms in charge of generating, removing, or transporting TKE. 
Unlike in the rest of the upper atmospheric layers, turbulence is permanently present and shapes the lowest part of the atmosphere, the so-called planetary boundary layer (PBL). A deep knowledge of the thermodynamics of the PBL is key to fulfil some of the main Martian science goals, namely: determine if life ever arose on Mars, understand the processes of climate, and prepare for human exploration (MEPAG, 2008). Addressing the TKE budget accordingly, by elucidating the weight of each of the mechanisms involved in it, becomes a first and necessary step to gain insight into the previous goals.

To date, little is known about the TKE on Mars. The reason lies in the nature of the measurements that should be done to investigate it. The TKE is defined as $\bar{e}=0.5\left(\overline{\sigma_{u}^{2}}+\overline{\sigma_{v}^{2}}+\overline{\sigma_{w}^{2}}\right)$, where the overbars over the wind speed variances denote averaging over periods of $30 \mathrm{~min}$ to one hour. This way, the departure from the mean corresponds to the turbulent part of the spectrum (Van der Hoven, 1957). Such averaging methodology is known as the Reynolds average form (Stull, 1988). Based on the TKE definition, and on the equation of the TKE budget described in detail in section 3, high-frequency and simultaneous measurements at several heights of temperature, horizontal and vertical wind speed, humidity, and pressure would in principle be required to study the TKE budget in the lowest part of the PBL (Wyngaard and Côté, 1971). Likewise, balloon or aircraft measurements should be carried out to perform an analogue study in the upper PBL (Kaimal et al., 1976). However, equipment capable of performing these kind of measurements still cannot be afforded by current technology and the financial budget. Thus, researchers have focused on creating numerical three-dimensional (3D) mesoscale models, and more specifically, large-eddy simulation (LES) models to study the turbulence in the Martian PBL.

Mars 3D mesoscale models are adaptations to Martian conditions of well-proven mesoscale Earth models. As far as we know, four terrestrial 3D mesoscale models have been adapted to Mars:

(i) the Regional Atmospheric Modeling System (RAMS; Pielke et al., 1992);

(ii) the Mesoscale Model Version 5 (MM5; Dudhia, 1993);

(iii) the Weather Research and Forecasting model (WRF; Skamarock et al., 2005); and

(iv) the High-Resolution Limited-Area Model (HIRLAM; Undén et al., 2002).

By properly increasing their resolution below the size of the eddies, though maintaining the total domain large enough to capture many of them (to allow representative statistics), and by taking as initial thermal forcings the 'outputs' of these 3D mesoscale models (among other adaptations), some LESs have been created and run for the Martian PBL. Together with LESs linked to 3D mesoscale models, pure idealized LESs have been applied to Mars. In both cases, flat terrain and cyclic boundary conditions are assumed.

We start by recapitulating LESs linked to 3D mesoscale models, emphasizing those results related to the TKE and its budget. Rafkin et al. (2001) created the seminal Martian LES by adapting the terrestrial RAMS model, creating MRAMS model. Vertical profiles of vertical velocity variance and TKE for an idealized Martian location were shown in this seminal work. MRAMS model was subsequently used by Michaels and Rafkin (2002), where a qualitative discussion about the
TKE was presented for the Pathfinder (PF) landing site. The next 3D model to be adapted to Mars was the terrestrial MM5 model, which was tuned by Toigo et al. (2003) to reproduce the formation of dust devils and their associated TKE budget on Terra Meridiani. The first adaptation to Mars of terrestrial WRF was done by Richardson et al. (2007), where a LES was run to show preliminary results at an idealized Martian location. A second adaptation, with different characteristics, was carried out by Spiga and Forget (2009), who showed vertical TKE profiles during the afternoon at the Mars Exploration Rover landing site Gusev Crater. (Useful information for the Gusev Crater turbulent environment is also provided by the single-column model shown by Savijärvi and Kauhanen, 2008). Via the same model, Spiga et al. (2010) ran five LESs at the same locations for which Hinson et al. (2008) calculated the PBL height, providing vertical TKE profiles. Finally, Kauhanen et al. (2008) adapted HIRLAM to Mars with the objective of preparing eventual analyses of the meteorological data returned by the Phoenix Lander, though no TKE results were shown.

Referring to the idealized LES-not linked to 3D models-Sorbjan(2007a, 2007b) performed three different simulations by changing the strength of the radiative heating, all of them at no specific Martian location. Vertical profiles of horizontal and vertical velocity variances were presented in non-dimensional form. Finally, supporting the Phoenix mission, Tyler et al. (2008) adapted to Mars the terrestrial Skyllingstad (2003) LES model, and showed vertical TKE profiles and a qualitative discussion of buoyancy and shear.

The two types of LES previously mentioned, undoubtedly among the most powerful tools to research the Martian PBL, have shown vertical TKE profiles, but without addressing the TKE budget, except for the dust devil case presented by Toigo et al. (2003).

In this article we present both innovative results and a new methodology. Values for the different mechanisms involved in the TKE budget equation will be derived for the Martian PBL during the most convective hours, both for the lowest surface layer (SL), and for the upper convectively driven mixed layer (CML) (Stull, 1988, gives their definitions). The comparative role of the storage term, buoyancy, shear, vertical turbulent transport, dissipation, and an imbalance term accounting for the remainder of the TKE budget will be determined in both layers. In addition, our study will be carried out from surface-based data corresponding to Viking and PF missions, and from an adaptation of similarity theory to Mars, which constitutes a new approach to investigate the TKE budget. Using this simple scheme-compared to the extremely time-expensive LES-some of our results have been found to compare well with already published LES outcomes, which increases our confidence in our new results and methodology.

Section 2 shows the data used as 'inputs', namely in situ (surface-based) horizontal wind speed and temperature, and modelled ground temperature. They all belong to some selected Sols (1 Sol is one Martian day, spanning 88775 s) of Viking Lander 1 (VL1), Viking Lander 2 (VL2) and PF missions. Section 3 explains the methodology, and is split into three subsections, describing the approach taken to study the SL TKE budget, the CML TKE budget, and the limitations of both approaches. Section 4 presents the results, and is also divided into three subsections: the TKE budget for the unstable SL, the TKE budget for the CML, and the 
sensitivity results to some key parameters. Section 5 gives a discussion of the results, and is formed of two subsections, the first addressing where the imbalance comes from, and the second comparing the results to those obtained under fairweather conditions and over flat terrain on Earth. Finally, the main conclusions are presented in section 6 .

\section{Data}

The set of data employed in this work consists of:

(i) in situ hourly-averaged temperature,

(ii) in situ hourly-averaged horizontal wind speed measured at the same height as the temperature, and

(iii) modelled hourly ground temperature, $T_{\mathrm{g}}$.

They all belong to three selected entire Martian Sols, namely Sol 25-26 of PF, Sol 28 of VL1, and Sol 20 of VL2. They correspond to solar longitudes $\mathrm{L}_{\mathrm{s}}=110^{\circ}, 127^{\circ}$ and $155^{\circ}$, respectively, where $L_{s}$ stands for the Mars-Sun angle, defined as starting from the northern spring equinox.

Though the hourly-averaged zonal and meridional wind speed are shown in the NASA Planetary Data System (PDS) for the VLs, we have here defined our $x$ axis to be aligned with the mean combined horizontal velocity, which we will simply name as $\bar{U}$. Following this assumption, $\bar{V}=0$. This convention is also valid for the PF mission and applies to the whole article, in both the SL and the CML.

We begin by describing the in situ data. For the Viking missions, VL1 touched down on 20 July 1976 , at $22.5^{\circ} \mathrm{N}$, $48^{\circ} \mathrm{W}$, and VL2 on 3 September the same year at $48.5^{\circ} \mathrm{N}$, $225^{\circ} \mathrm{W}$. It was summertime at both locations, corresponding to $L_{S}=98^{\circ}$ and $120^{\circ}$, respectively. Viking missions measured pressure, temperature, and horizontal wind speed at $1.6 \mathrm{~m}$ height over 3.3 Mars years in the case of VL1, and over 1.6 Mars years in the case of VL2 (Hess et al., 1977).

Among all the available Viking Sols, we have selected VL1 Sol 28 and VL2 Sol 20 because only during these were some indispensable conditions guaranteeing the accuracy of the measurements met (Sutton et al., 1978). At both sites, and through these Sols, the prevailing wind direction involved the least interference from the lander. Wind speed and temperature sampling rate during the strongest convective hours were very high (up to $2 \mathrm{~Hz}$ ) compared with the rest of the mission, which implied more accurate means and variances. The accuracy of the sensors was at their highest level at that time for both missions. Since sampling took place during the northern summertime, two additional requirements were met: baroclinic disturbances (which could result in the mixed layer not being convectively driven, and therefore a worse performance of the adapted similarity theory) were not present, and the amount of atmospheric dust (which could also hinder its performance) was at a minimum.

In Figure 1 we show hourly averaged temperature and horizontal wind speed for VL1 Sol 28 and VL2 Sol 20. These averaged measurements, together with their sampling rates, are available in the NASA PDS.

Turning to the PF mission, it touched down on 4 July 1997 at $19.7^{\circ} \mathrm{N}, 33.55^{\circ} \mathrm{W}$ in the Ares Vallis region of Chryse Planitia. It was northern summer at that time, corresponding to $\mathrm{L}_{\mathrm{s}}=140^{\circ}$. Through the ASI/MET package (Schofield et al., 1997; Wilson and Joshi, 2000), the PF mission monitored

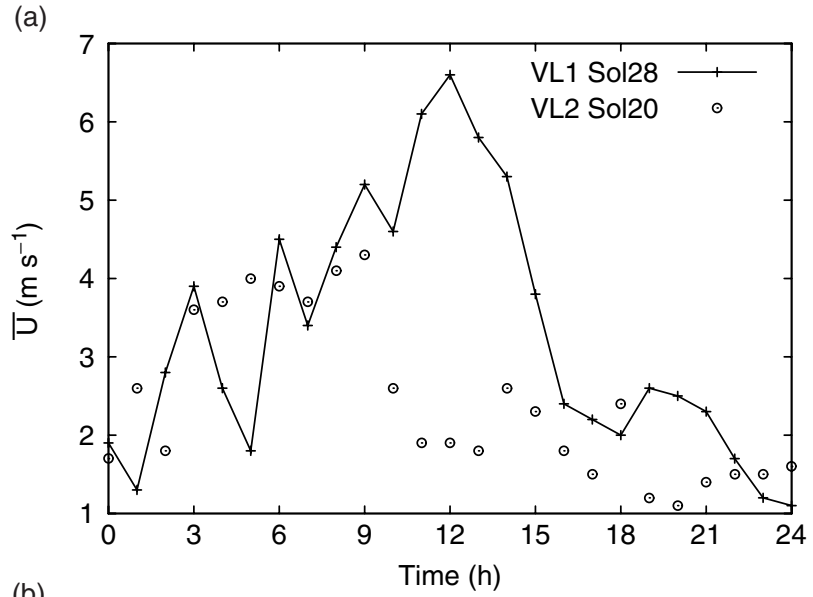

(b)

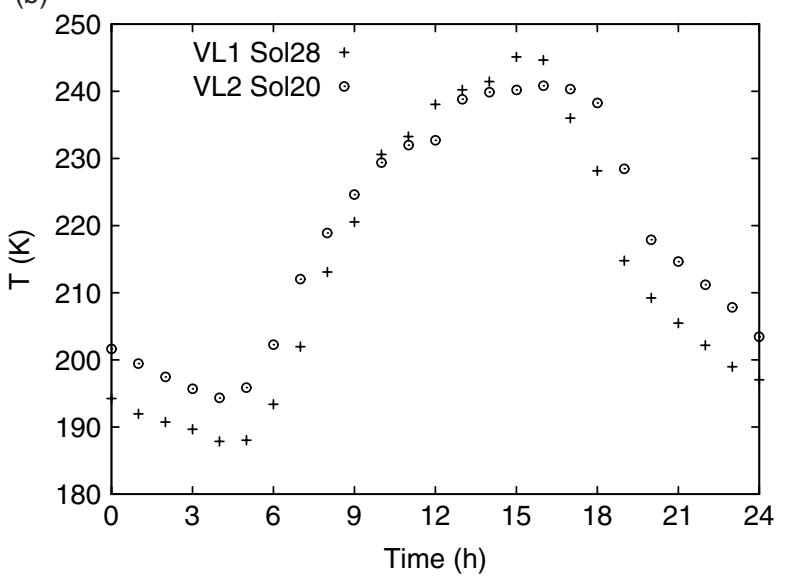

Figure 1. VL1 Sol 28 and VL2 Sol 20 hourly averaged values of (a) horizontal wind speed and (b) temperature, both measured at $1.6 \mathrm{~m}$ at both locations, and available in the NASA PDS. In (a), wind speed for VL1 Sol 28 is shown as a solid line for clarity, although the values are still discrete.

temperature at three heights $(1.27 \mathrm{~m}, 0.77 \mathrm{~m}$, and $0.52 \mathrm{~m}$ above the ground), horizontal wind speed at a single height ( $1.30 \mathrm{~m}$ above the ground), and pressure at a single height for 83 Sols. Continuous sampling for a complete Sol was conducted just on Sols 25-26, 32, 38, 55, and 68. However, we note that the PF wind sensor experienced problems related to the overheating of the sensor wire segments from which the wind speed was derived. Therefore just temperature and pressure data can be found in the NASA PDS.

However, we were given quality-controlled horizontal wind speed data (J. Murphy, New Mexico University, personal communication) covering the period from 0600 Sol 25 to 0600 Sol 26. These data have already been used by Savijärvi et al. (2004), Määttänen and Savijärvi (2004), and Martinez et al. (2009a). Both the derived horizontal velocity and the PDS temperature were monitored at a $0.25 \mathrm{~Hz}$ rate for the same period of time. Hourly averaged values of temperature and horizontal wind speed for PF Sol 25-26 are shown in Figure 2.

With regard to the simulated data, we have derived $T_{\mathrm{g}}$ values for the three selected Sols by running a modification of the 1D microscale model of the University of Helsinki shown in Savijärvi et al. (2004). Since ground temperature is a key parameter driving the PBL, and was not obtained in situ, special rigour has been applied to calculate it. For each Sol, we have simulated three $T_{\mathrm{g}}$ scenarios: the most probable scenario, shown in Figure 3, and the warmest and the coldest scenarios, which represent reliable extreme scenarios 


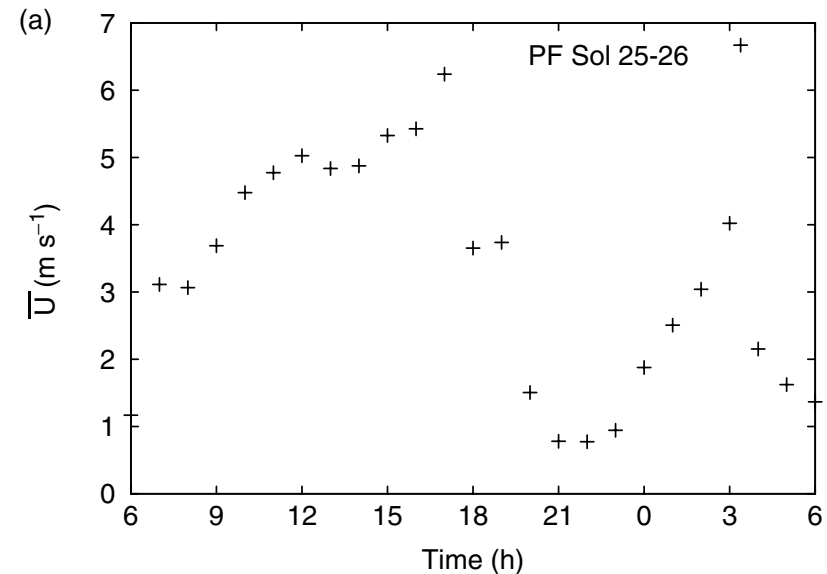

(b)

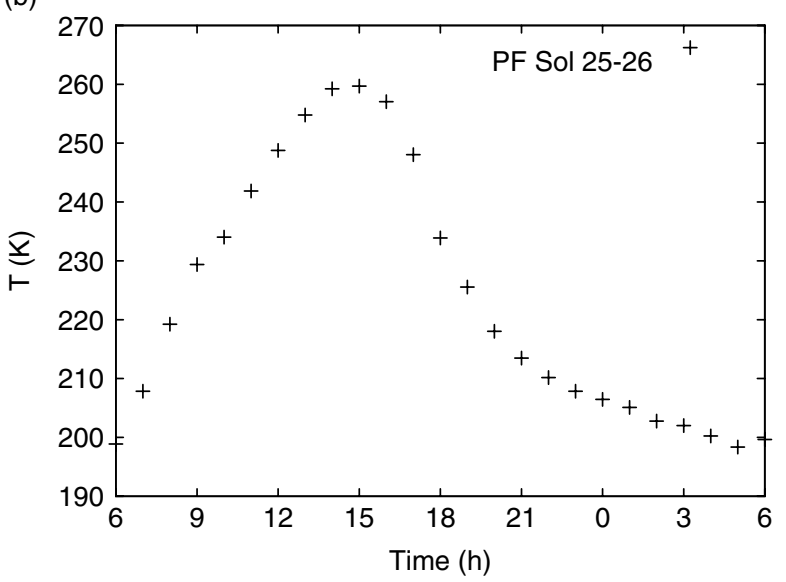

Figure 2. PF Sol 25-26 hourly averaged values of (a) horizontal wind speed and (b) temperature, measured at 1.3 and $1.27 \mathrm{~m}$, respectively. Hourly averaged temperature values are available in the NASA PDS, but speed data are not.

of ground temperature; these have been used to perform sensitivity studies, as will be shown in subsection 4.3. More details about the procedure used to derive these scenarios are given in Martinez et al. (2009b).

\section{Methodology}

The equation governing the evolution of the TKE in the Reynolds average form, under the assumptions of Newtonian fluid, Boussinesq approximation, and Einstein notation (the indices $i$ and $j$ take the values 1,2 , or 3 corresponding respectively to the $x, y$ and $z$ axis, as explained in Stull, 1988) can be written as

$$
\begin{aligned}
\frac{\partial \bar{e}}{\partial t}= & -\overline{U_{j}} \frac{\partial \bar{e}}{\partial x_{j}}+\delta_{i 3} \frac{g}{\bar{\theta}} \overline{u_{i}^{\prime} \theta^{\prime}}-\overline{u_{i}^{\prime} u_{j}^{\prime}} \frac{\partial \bar{U}_{i}}{\partial x_{j}} \\
& -\frac{\partial \overline{u_{j}^{\prime} e}}{\partial x_{j}}-\frac{1}{\bar{\rho}} \frac{\partial \overline{u_{j}^{\prime} p^{\prime}}}{\partial x_{j}}-\epsilon,
\end{aligned}
$$

where $\overline{U_{j}}$ denotes the mean wind, $u_{j}^{\prime}$ the turbulent deviation of the velocity, $\theta^{\prime}$ the turbulent deviation of the potential temperature, $\bar{\rho}$ the mean air density, $p^{\prime}$ the turbulent deviation of pressure, and $\epsilon$ the dissipation term, or the conversion of TKE into heat by molecular viscosity.

Equation (1) states that TKE can vary (the storage term on the left-hand side) as a consequence of different mechanisms shown on the right-hand side. It can be advected by
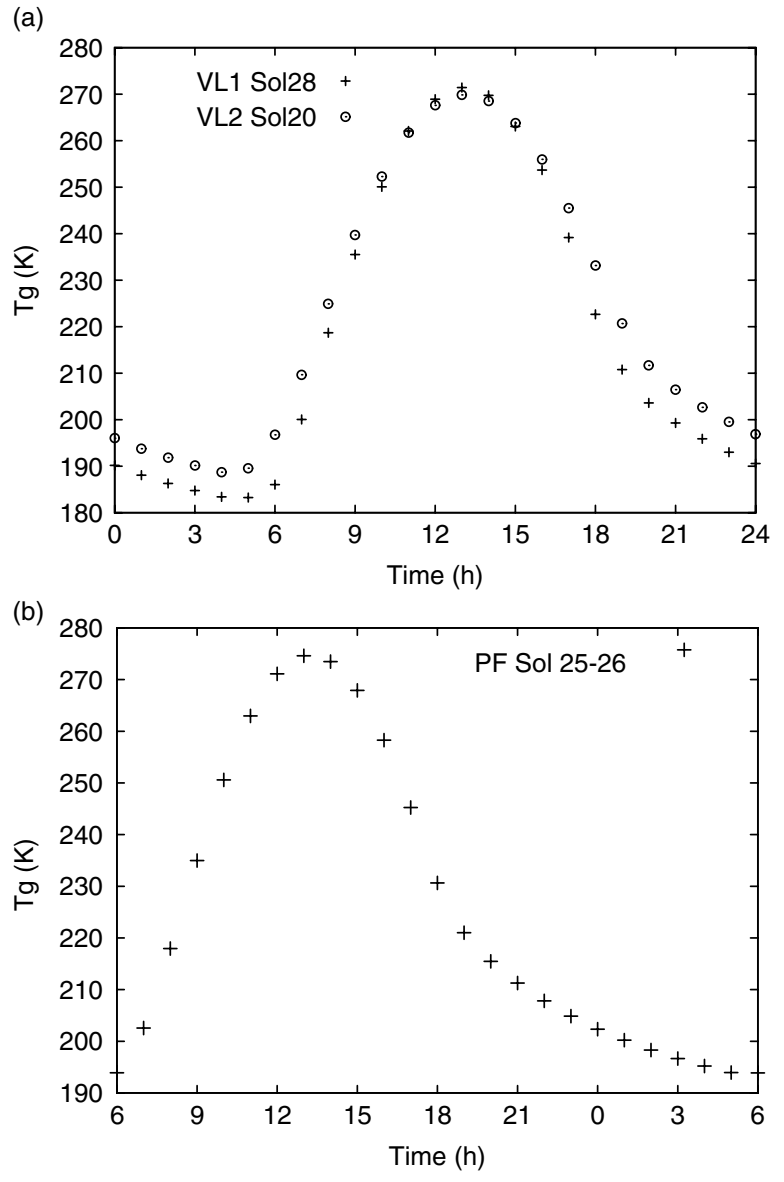

Figure 3. Ground temperature of the most probably scenario for (a) VL1 Sol 28 and VL2 Sol 20, and for (b) PF Sol 25-26.

the mean wind, generated or destroyed by buoyancy and shear, transported by the turbulent transport term and the turbulent pressure redistribution term, and finally destroyed via molecular dissipation, respectively. Equation (1) can be rewritten for convenience as

$$
\begin{aligned}
0 & =-\frac{\partial \bar{e}}{\partial t}+\frac{g}{\bar{\theta}} \overline{w^{\prime} \theta^{\prime}}-\overline{u^{\prime} w^{\prime}} \frac{\partial \bar{U}}{\partial z}-\frac{\partial \overline{w^{\prime} e}}{\partial z}-\epsilon+I m \\
& =-S t+B u+S h+T r-D i s s+I m
\end{aligned}
$$

where $S t$ denotes the storage term, $B u$ the buoyancy, Sh the vertical shear term, $\operatorname{Tr}$ the vertical (main) turbulent transport, Diss the dissipation, and Im the remainder of the TKE budget terms, which we have grouped into one term called imbalance, named as in Wyngaard and Côté (1971) and written as

$$
\begin{aligned}
I m= & -\bar{U} \frac{\partial \bar{e}}{\partial x}-\bar{W} \frac{\partial \bar{e}}{\partial z}-\frac{1}{\bar{\rho}} \frac{\partial \overline{u_{j}^{\prime} p^{\prime}}}{\partial x_{j}}-\frac{\partial \overline{u^{\prime} e}}{\partial x}-\frac{\partial \overline{v^{\prime} e}}{\partial y} \\
& -\overline{u^{\prime} 2} \frac{\partial \bar{U}}{\partial x}-\overline{w^{\prime 2}} \frac{\partial \bar{W}}{\partial z}-\overline{u^{\prime} w^{\prime}} \frac{\partial \bar{W}}{\partial x}-\overline{u^{\prime} v^{\prime}} \frac{\partial \bar{U}}{\partial y} \\
& -\overline{v^{\prime} w^{\prime}} \frac{\partial \bar{W}}{\partial y}
\end{aligned}
$$

where the first two terms account for the advection, the third for pressure redistribution, the fourth and the fifth for the horizontal transport of TKE, and the remainder 
Table I. Key SL magnitudes: Monin-Obukhov length $L$, friction velocity $u_{*}$, and scale temperature $T_{*}$. The values shown denote the average over the most unstable hours of each mission.

\begin{tabular}{lccc}
\hline & VL1 Sol 28 & VL2 Sol 20 & PF Sol 25 \\
\hline$L(\mathrm{~m})$ & -40 & -7 & -25 \\
$u_{*}\left(\mathrm{~m} \mathrm{~s}^{-1}\right)$ & 0.5 & 0.25 & 0.4 \\
$T_{*}(\mathrm{~K})$ & -1.5 & -2.0 & -1 \\
\hline
\end{tabular}

for the original shear term $\overline{u_{i}^{\prime} u_{j}^{\prime}} \partial \bar{U}_{i} / \partial x_{j}$, except for the already extracted $\overline{u^{\prime} w^{\prime}} \partial \bar{U} / \partial z$ term. Note that it has been assumed that $\bar{V}=0$. To avoid misunderstanding, the vertical shear term $-\overline{u^{\prime} w^{\prime}} \partial \bar{U} / \partial z$ will be simply called shear, while horizontal shear will be used for the remaining shear terms.

From the data described in section 2, and via an adaptation to Mars of similarity theory, we will obtain values for each of the terms appearing in Eq. (2), except for Im, which will be obtained precisely as the remainder needed to balance this equation. This is one of the reasons for which Eq. (1) has been rearranged into Eq. (2). The other reason is that the term $I m$ is expected to have a non-prominent role under ideal conditions for the application of similarity theory, as will be shown in next sections.

In the next two subsections, we describe the methodology used to determine values for each of the TKE budget terms in Eq. (2), both in the SL and in the CML, respectively. The limitation of the methodology will be clarified in the third subsection.

\subsection{Surface layer approach}

An adaptation of terrestrial SL similarity theory (Monin and Obukhov, 1954) to Mars is used to derive the values for each of the terms in Eq. (2) within the SL. Such adaptation consists of coupling a molecular sublayer (i.e. the layer where the transfer of momentum and heat is dominated by molecular processes) to SL similarity theory, which in its standard terrestrial version neglects molecular diffusion compared with turbulent transport. Yet on Mars, molecular kinematic viscosity and thermal diffusivity are two orders of magnitude higher than on Earth $\left(10^{-3}\right.$ versus $\left.10^{-5} \mathrm{~m}^{2} \mathrm{~s}^{-1}\right)$. Therefore, only two orders of magnitude separate molecular diffusion from turbulent transport (typically $10^{-1} \mathrm{~m}^{2} \mathrm{~s}^{-1}$ ) in the first Martian metres, while for the terrestrial case, this difference typically grows to six orders of magnitude at these heights. Thus, on Mars, unlike on Earth, the need for including a molecular sublayer becomes evident.

This 'extended' SL similarity theory scheme is explained in detail in Martinez et al. (2009a), where it is applied to the PF location taking as inputs the same PF data presented in section 2 . The results shown in that article are some hourly derived key SL magnitudes shown in Table I, from which it is straightforward to obtain the TKE budget terms, as we next substantiate.

It is important to notice that the following equations, from which each TKE budget term will be obtained, are well-proven SL terrestrial similarity relationships, which have been derived over flat terrain, and under fair-weather conditions (no baroclinic disturbances). In subsection 3.3, it will be explained why these relations are also expected to work on Mars.

\subsubsection{Storage term}

The storage term $S t=\partial \bar{e} / \partial t$ can be obtained by means of finite differences from the temporal evolution of the TKE, expressed in this case as

$$
\bar{e}=\frac{1}{2}\left(\overline{\sigma_{u}^{2}}+\overline{\sigma_{w}^{2}}\right)
$$

where $\overline{\sigma_{u}}$ refers to the horizontal velocity standard deviation, and $\overline{\sigma_{w}}$ to the vertical component. The first magnitude can be calculated from the $0.25 \mathrm{~Hz}$ horizontal wind speed data for the PF Sol 25, while for the VL1 Sol 28 and VL2 Sol 20, it is found in the NASA PDS. On the other hand, the vertical velocity standard deviation $\overline{\sigma_{w}}$ during the most convective hours were derived for these three Sols, and from the same data, by Martinez et al. (2009b).

\subsubsection{Buoyancy term}

The buoyancy term $B u=(g / \bar{\theta}) \overline{w^{\prime} \theta^{\prime}}$ can be rewritten as

$$
B u=-\frac{g}{T_{\mathrm{g}}} u_{*} T_{*},
$$

where the turbulent heat flux $\overline{w^{\prime} \theta^{\prime}}$ at 1.6 and $1.3 \mathrm{~m}$ should be virtually equal to its value at the ground $\left(\overline{w^{\prime} \theta^{\prime}} \simeq \overline{w^{\prime} \theta^{\prime}}{ }_{s}\right)$, this last magnitude being in turn equal to $-u_{*} T_{*}$.

\subsubsection{Shear term}

The shear term $S h=-\overline{u^{\prime} w^{\prime}} \partial \bar{U} / \partial z$ can be expressed as

$$
S h=\frac{u_{*}^{3}}{k z} \phi_{m}\left(\frac{z}{L}\right),
$$

with $-\overline{u^{\prime} w^{\prime}} \simeq-\overline{u^{\prime} w_{s}^{\prime}} \simeq u_{*}^{2}, \partial \bar{U} / \partial z=\left(u_{*} / k z\right) \phi_{m}(z / L)$, and $\phi_{m}(z / L)$ the universal function for momentum defined as in Högström (1988). Note that the turbulent momentum flux $\overline{u^{\prime} w^{\prime}}$ at 1.6 and $1.3 \mathrm{~m}$ has been considered constant and equal to the value at the surface, since the SL is a constant flux layer.

\subsubsection{Main turbulent transport term}

The main (vertical) turbulent transport term $T r=$ $-\partial \overline{w^{\prime} e} / \partial z$ can be rewritten as

$$
\operatorname{Tr}=2.3 \frac{u_{*}^{3}}{L},
$$

where the vertical turbulent transport of TKE during the most convective hours can be expressed as $\overline{w^{\prime} e}=-2.3 u_{*}^{3}(z / L)$, based on results obtained by Wyngaard and Côté (1971) and Lenschow et al. (1980).

\subsubsection{Dissipation term}

Finally, and also during the most convective daytime hours, the dissipation term $\epsilon$ can be obtained as

$$
\epsilon=\frac{u_{*}^{3}}{k z}\left(1+0.5\left|\frac{z}{L}\right|^{2 / 3}\right)^{3 / 2},
$$


Table II. Key CML magnitudes: PBL height $z_{\mathrm{i}}$, and the convective scaling velocity $w_{*}$. The values shown denote the average over the most unstable hours of each mission.

\begin{tabular}{lccc}
\hline & VL1 Sol 28 & VL2 Sol 20 & PF Sol 25 \\
\hline$z_{\mathrm{i}}(\mathrm{km})$ & 10 & 2 & 7 \\
$w_{*}\left(\mathrm{~m} \mathrm{~s}^{-1}\right)$ & 4 & 2 & 4.4 \\
\hline
\end{tabular}

as shown in Martinez et al. (2009a) for Mars, although the equation was originally found for Earth by Wyngaard and Côté (1971).

\subsection{Convective mixed-layer approach}

The approach taken to obtain values for the TKE budget in the Martian CML is analogue to the SL approach. An adaptation of terrestrial CML similarity theory (Deardorff, 1972) to Mars is used to derive the values for each term in Eq. (2) within the CML. Details of this adaptation (molecular sublayer also coupled) appear in Appendix C of Martinez et al. (2009b), where it is applied to the VL1, VL2, and PF locations, taking as 'inputs' the same data as presented in section 2. As for the SL, the results shown in that article are some hourly derived key CML magnitudes shown in Table II, from which the CML TKE budget terms can be obtained, as it is shown in this subsection.

Due to the nature of our 'inputs', which were taken at a single height within the SL, only averaged values for the CML TKE budget terms can be derived (instead of vertical profiles). Thus, we define the CML average of any magnitude $\xi(z, t)$ as

$$
\langle\xi(z, t)\rangle=\frac{1}{0.8 z_{\mathrm{i}}-0.2 z_{\mathrm{i}}} \int_{0.2 z_{\mathrm{i}}}^{0.8 z_{\mathrm{i}}} \xi(z, t) \mathrm{d} z,
$$

where the limits of the integral enclose the bulk of the CML. (Note that, although not explicitly written, the height of the CML, $z_{\mathrm{i}}$, varies with time.) Across these limits, the applicability of the adapted CML similarity theory is more accurate, since the entrainment zone and the SL (where the thermodynamic characteristics are different) evolve well above and below such limits, respectively. Following Eq. (9), Eq. (2) converts in the CML into

$$
0=-\langle S t\rangle+\langle B u\rangle+\langle S h\rangle+\langle T r\rangle-\langle\text { Diss }\rangle+\langle\operatorname{Im}\rangle .
$$

We next demonstrate how each term of Eq. (10) can be derived from $z_{\mathrm{i}}$ and $w_{*}$. As for the $\mathrm{SL}$, the next equations shown are proven terrestrial CML similarity relationships, derived for flat terrain and under specific atmospheric conditions. Only the equation for buoyancy has not been taken from terrestrial similarity relationships, but from Martian studies. However, it closely matches the analytical terrestrial form, as shown in Figure 11 in Spiga et al. (2010).

\subsubsection{Storage term}

The averaged storage term can be expressed as

$$
\left\langle\frac{\partial \bar{e}}{\partial t}\right\rangle=\frac{\partial\langle\bar{e}\rangle}{\partial t}+\frac{1}{z_{\mathrm{i}}} \frac{\partial z_{\mathrm{i}}}{\partial t}\left\{\langle\bar{e}\rangle+\frac{1}{3} \bar{e}\left(0.2 z_{\mathrm{i}}\right)-1.3 \bar{e}\left(0.8 z_{\mathrm{i}}\right)\right\},
$$

where the Leibnitz rule and Eq. (9) have been used. The term $\partial\langle\bar{e}\rangle / \partial t$ can be obtained by means of finite differences from the temporal evolution of the averaged TKE, expressed in this case as

$$
\langle\bar{e}\rangle=\frac{1}{2}\left(\left\langle\overline{\sigma_{u}^{2}}\right\rangle+\left\langle\overline{\sigma_{w}^{2}}\right\rangle\right)
$$

where the horizontal $\left\langle\overline{\sigma_{u}}\right\rangle$ and vertical $\left\langle\overline{\sigma_{w}}\right\rangle$ averaged velocity standard deviations were derived by Martinez et al. (2009b) based on terrestrial similarity relationships shown in Stull (1988). In addition, and also based on results shown by Martinez et al. (2009b), it can be assumed as typical values that $z_{\mathrm{i}} \simeq 5 \times 10^{3} \mathrm{~m},\langle\bar{e}\rangle \simeq \bar{e}\left(0.2 z_{\mathrm{i}}\right) \simeq \bar{e}\left(0.8 z_{\mathrm{i}}\right) \simeq 5 \mathrm{~m}^{2} \mathrm{~s}^{-2}$, and $\partial z_{\mathrm{i}} / \partial t \leq 10^{-1} \mathrm{~m} \mathrm{~s}^{-1}$, thus all the terms forming the averaged storage term are known. We point out that, based on Sorbjan et al. (2009), the previously given value for $\partial z_{\mathrm{i}} / \partial t$ represents an upper bound during the most unstable hours, since this value corresponds to the early morning hours, when the rate of increase of $z_{\mathrm{i}}$ is the highest. Therefore, the values for the CML storage term displayed in next sections are upper bounds. Even so, their impact on the CML TKE budget is negligible, as will be shown.

\subsubsection{Buoyancy term}

We start with the buoyancy term $\langle B u\rangle=\left\langle(g / \theta) \overline{w^{\prime} \theta^{\prime}}\right\rangle$. The turbulent heat flux $\overline{w^{\prime} \theta^{\prime}}$ can be expressed at any height in the range $0.2 z_{\mathrm{i}}-0.8 z_{\mathrm{i}}$ as

$$
\overline{w^{\prime} \theta^{\prime}}=\frac{\bar{\theta}}{g} \frac{w_{*}^{3}}{z_{\mathrm{i}}}\left(1.2-1.5 \frac{z}{z_{\mathrm{i}}}\right)
$$

derived from Spiga et al. (2010). In fact, Spiga et al. (2010) obtained Eq. (13) for $\overline{w^{\prime} \theta^{\prime}}$, with the only difference that $w_{*}$ was substituted by $W_{*}^{\max }$, where

$$
W_{*}^{\max }=\left(\frac{g}{\bar{\theta}} z_{\mathrm{i}} \overline{w^{\prime} \theta^{\prime}} \max \right)^{1 / 3} .
$$

However, given the low radiative heating present during our Sols, $\overline{w^{\prime} \theta^{\prime}} \max / \overline{w^{\prime} \theta^{\prime}}{ }_{s}<1.4$ is satisfied, which implies that $W_{*}^{\max } / w_{*}<1.12$. Thus, under low radiative heating, the work of Spiga et al. (2010) also supports the use of Eq. (13).

The averaged buoyancy term can be eventually written as

$$
\langle B u\rangle=\frac{1}{0.6 z_{\mathrm{i}}} \frac{w_{*}^{3}}{z_{\mathrm{i}}} \int_{0.2 z_{\mathrm{i}}}^{0.8 z_{\mathrm{i}}}\left(1.2-1.5 \frac{z}{z_{\mathrm{i}}}\right) \mathrm{d} z .
$$

\subsubsection{Shear term}

We turn to describe the shear term $\langle S h\rangle=\left\langle-\overline{u^{\prime} w^{\prime}}(\partial \bar{U} / \partial z)\right\rangle$. Assuming a linear decrease of $\overline{u^{\prime} w^{\prime}}$, shear can be obtained as

$$
\langle S h\rangle=\frac{1}{0.6 z_{\mathrm{i}}} \frac{w_{*}^{3}}{z_{\mathrm{i}}} \int_{0.2 z_{\mathrm{i}}}^{0.8 z_{\mathrm{i}}}\left(\frac{z}{z_{\mathrm{i}}}-1\right) \frac{L}{z} \phi_{m}\left(\frac{z}{L}\right) \mathrm{d} z,
$$

where we have taken advantage of the study by Lenschow (1974). It is important to notice that the assumed variation in height for $\overline{u^{\prime} w^{\prime}}$ has very little effect on the results, even when it is considered constant. 


\subsubsection{Main turbulent transport term}

The vertical turbulent transport of TKE can be expressed in the bulk of the CML as

$$
\overline{w^{\prime} e}=0.8 w_{*}^{3} \frac{z}{z_{\mathrm{i}}}\left(1-0.9 \frac{z}{z_{\mathrm{i}}}\right)^{2},
$$

where the work presented by Lenschow et al. (1980) has been used. Thus, the averaged vertical turbulent transport term converts into

$$
\langle T r\rangle=\frac{1}{0.6 z_{\mathrm{i}}} \int_{0.2 z_{\mathrm{i}}}^{0.8 z_{\mathrm{i}}}-\frac{\partial \overline{w^{\prime} e}}{\partial z} \mathrm{~d} z,
$$

where the derivative of Eq. (16) must be incorporated into Eq. (17).

\subsubsection{Dissipation term}

The averaged dissipation term $\langle\epsilon\rangle$ is obtained as

$$
\langle\epsilon\rangle=\frac{1}{0.6 z_{\mathrm{i}}} \frac{w_{*}^{3}}{z_{\mathrm{i}}} \int_{0.2 z_{\mathrm{i}}}^{0.8 z_{\mathrm{i}}} \frac{1}{2} \mathrm{~d} z,
$$

as pointed out by Martinez et al. (2009b). Note that the dissipative term is expected to be constant with height across the bulk of the PBL, and therefore its averaged value should coincide with the single value at any height in the range $0.2 z_{\mathrm{i}}-0.8 z_{\mathrm{i}}$.

\subsection{Limitations}

The methodology, and consequently the results derived from it, are reliable provided that the following conditions are met:

(i) VLs and PF landing sites are moderately flat,

(ii) there are no baroclinic disturbances, and

(iii) there exists low radiative heating compared to the turbulent one across the CML (low dust content).

The more these assumptions are met, the more guarantees we have for the correct performance on Mars of Eqs (5), (6), (7), and (8) in the SL, and Eqs (14), (15), (17), and (18) in the CML.

Martínez et al.(2009a, 2009b) indicate the limitations of the SL and CML similarity theory applied to Mars, and the implied uncertainty in applying the above equations to Mars. It can be checked in these articles that the moderately flat terrain for VL and PF sites, and the specific atmospheric conditions (weak radiative heating and no baroclinic disturbances) existed especially during VL1 Sol 28 and PF Sol 25, and to a lesser extent during VL2 Sol 20 , to allow for the proper performance of the described methodology.

\section{Results}

The results are arranged into three subsections. The first one shows values for the TKE itself and for its budget in the Martian SL. The second subsection does the same, but for the Martian CML. The third subsection shows sensitivity results for the three different ground temperature scenarios, the height of the surface roughness, and the effect of not including a molecular sublayer in our adaptation to Mars of terrestrial similarity theory.
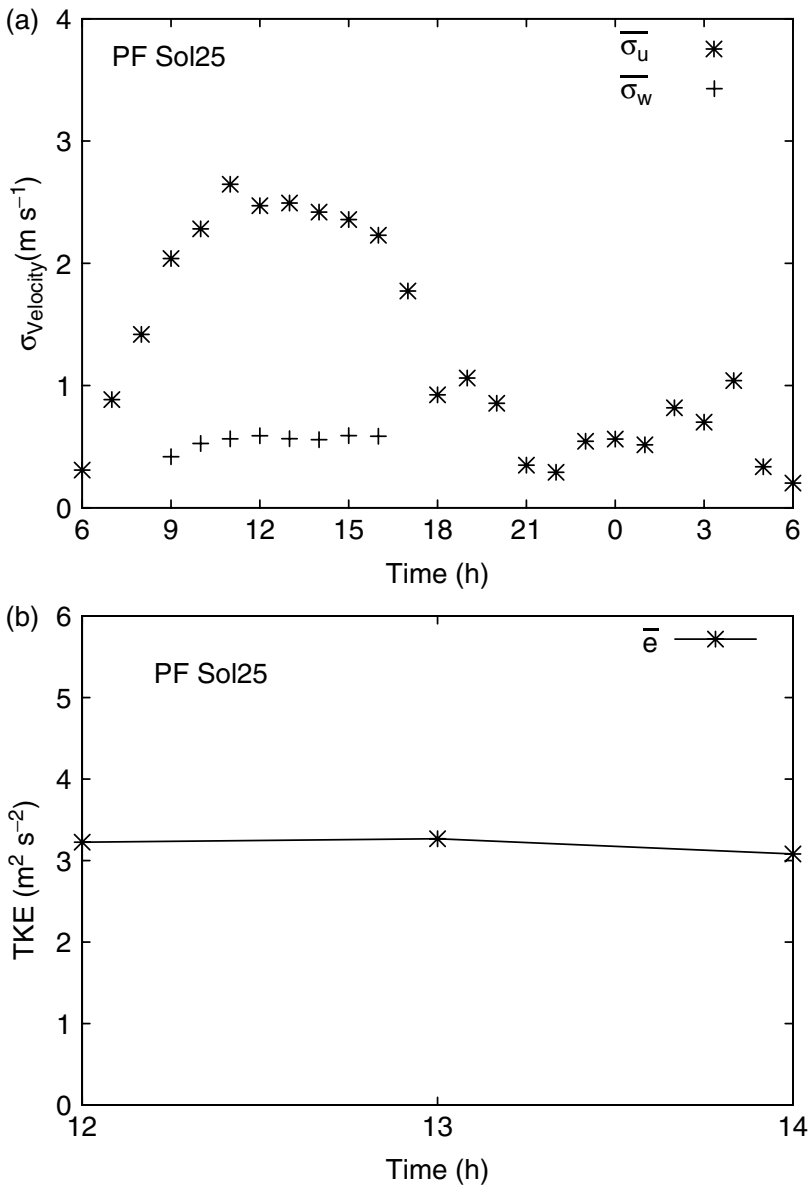

Figure 4. PF Sol 25-26 surface layer statistics: (a) hourly values for horizontal $\overline{\sigma_{u}}$ and vertical $\overline{\sigma_{w}}$ velocity standard deviations, and (b) TKE obtained from these at the most convective hours. These magnitudes have been estimated at $1.3 \mathrm{~m}$. TKE is shown as a solid line for clarity, though the estimations are discrete.

\subsection{TKE budget in the surface layer}

For the PF Sol 25, VL1 Sol 28, and VL2 Sol 20, the SL results are arranged as follows: the horizontal standard deviation $\overline{\sigma_{u}}$, together with the vertical component $\overline{\sigma_{w}}$, and then the TKE derived from these. Finally, the budget for the TKE is described.

\subsubsection{PF Sol 25}

Figure 4 displays the velocity standard deviations, as well as the TKE calculated from these. The horizontal turbulence is observed to be around four times more intense than the vertical component close to noon, mostly because measurements were taken close to the ground, which implied that the vertical turbulence was constrained. On the other hand, TKE values are approximately constant (steady state), around $3 \mathrm{~m}^{2} \mathrm{~s}^{-2}$.

We now describe the SL TKE budget as expressed in Eq. (2). It is important to note that both the SL and CML TKE budgets will be displayed from $1200 \mathrm{~h}$ to $1400 \mathrm{~h}$ (local solar time), coinciding with the hours when turbulence should be totally developed, and when the methodology explained in section 3 performs more accurately.

Figure 5 shows the TKE budget for PF Sol 25, and exhibits the following features. Shear is the main source 


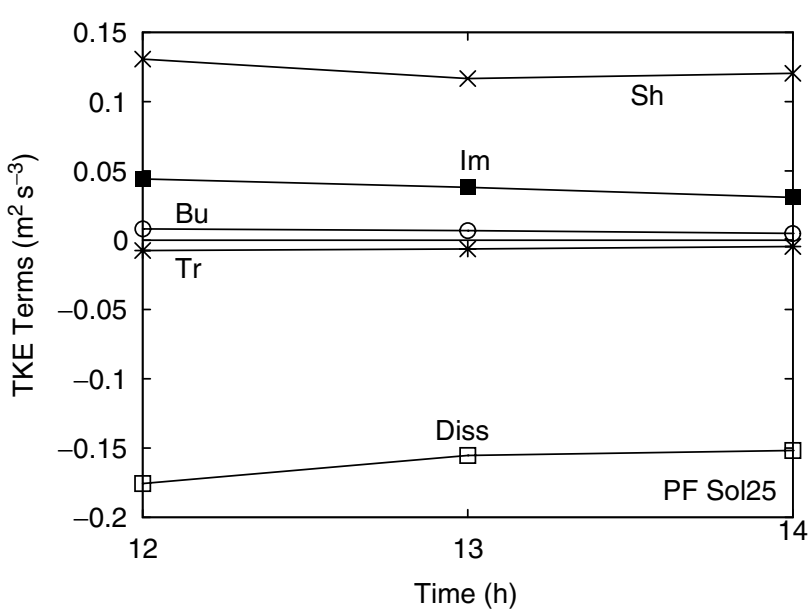

Figure 5. TKE budget in the surface layer for PF Sol 25, estimated at $1.3 \mathrm{~m}$. The results span the most convective hours (from $1200 \mathrm{~h}$ to $1400 \mathrm{~h}$, local solar time), when the TKE is expected to be totally developed. $B u$, $S h, T r$, Diss, and Im denote buoyancy, shear, vertical turbulent transport, dissipation, and the imbalance terms, respectively. The storage term $S t$ has not been included due to its negligible relevance, since its values are of the order of $10^{-5} \mathrm{~m}^{2} \mathrm{~s}^{-3}$, i.e. three orders of magnitudes less than the smallest $B u$ or $\operatorname{Tr}$ terms.

of TKE-expected at heights close to the ground-whereas dissipation is the main remover of TKE, barely exceeding the shear production. Both mechanisms present values of the order of $10^{-1} \mathrm{~m}^{2} \mathrm{~s}^{-3}$. Buoyancy and vertical turbulent transport balance each other, and are one order of magnitude lower than shear and dissipation. Thus, their roles on the TKE budget are not prominent. Specifically, buoyancy is positive (generation of TKE) since the surface kinematic heat flux was directed upwards close to noon. Vertical turbulent transport is negative, which indicates that it is removing TKE from the SL by sending it upwards. The imbalance term, though higher than the minor buoyancy and transport terms, is still about $30 \%$ of the ruling shear and dissipation mechanisms. It shows positive values, tending to balance the excess of destruction (dissipation) compared to generation (shear).

\subsubsection{VL1 Sol 28}

The velocity standard deviations and the TKE are shown in Figure 6. The results are quite similar to those found for PF Sol 25. The intensity of the turbulence on the horizontal plane is four times higher than in the vertical one during the most convective hours, and the TKE remains almost constant, presenting values close to $4 \mathrm{~m}^{2} \mathrm{~s}^{-2}$.

The SL TKE budget for VL1 Sol 28 is shown in Figure 7, where it can be observed that all the terms exhibit similar values to those for PF Sol 25. Shear is the dominant mechanism generating TKE, whereas dissipation is the principal remover. Buoyancy and vertical turbulent transport have little impact on the budget, and balance each other. The imbalance is somewhat higher than the minor buoyancy and vertical turbulent transport terms, and is about $30 \%$ of the main shear and dissipation mechanisms. Finally, the storage term plays an inconsequential role, with the same values as for the PF Sol 25, and thus is not included in the figure.

It is relevant to notice that our results for VL1 Sol 28 and PF Sol 25, despite relating to two different Martian locations and
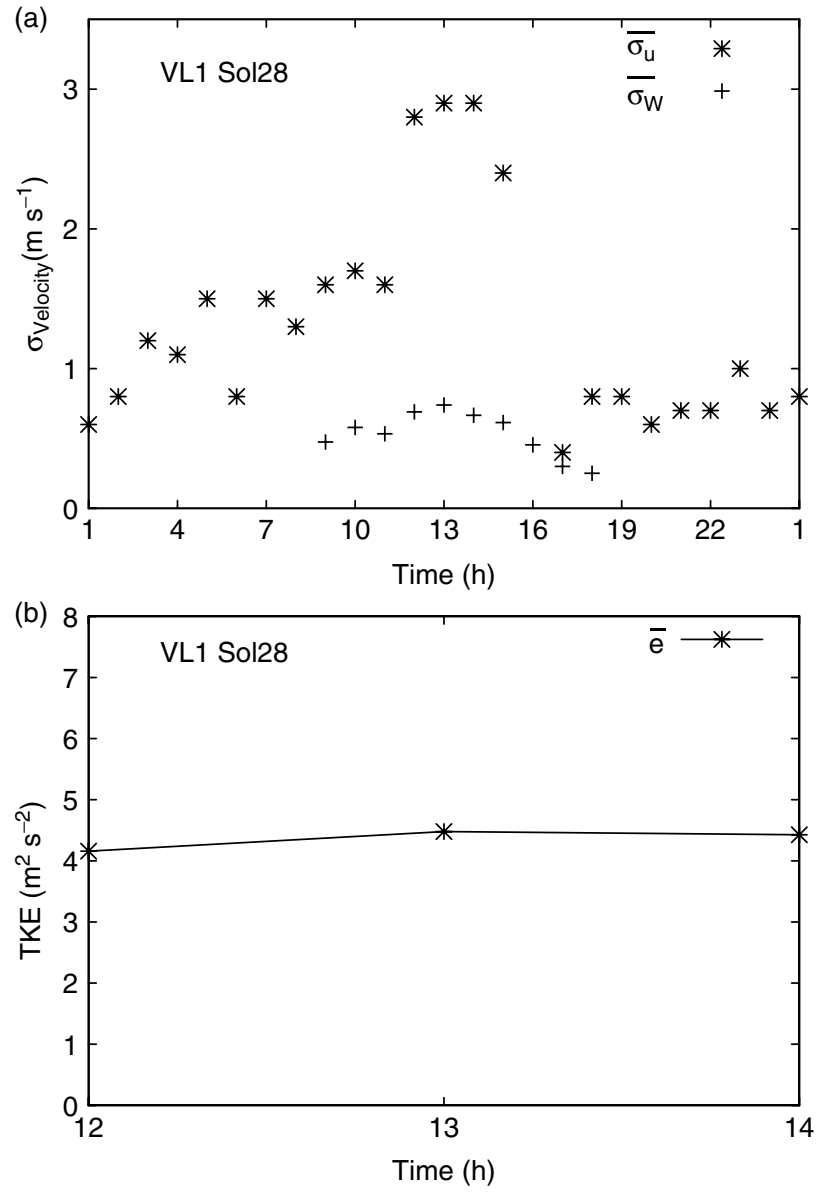

Figure 6. As Figure 4, but for VL1 Sol 28 at $1.6 \mathrm{~m}$.

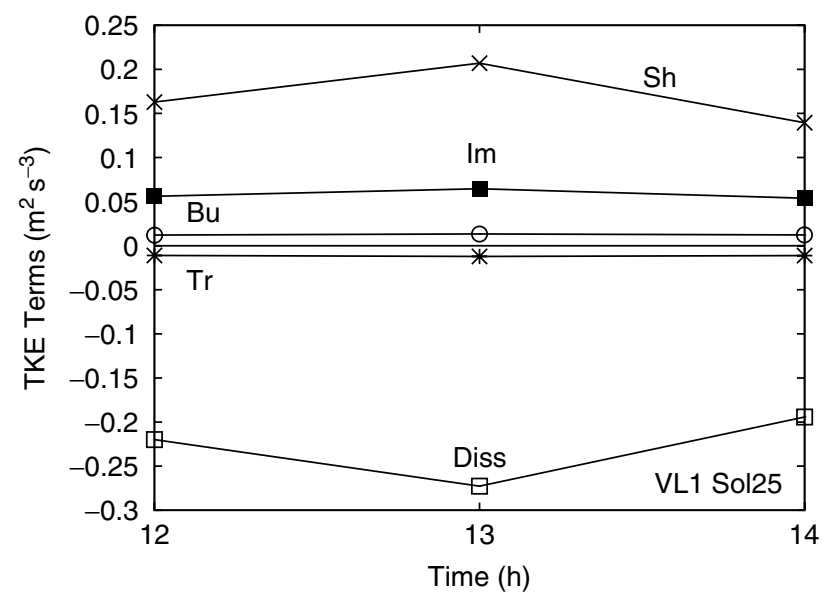

Figure 7. As Figure 5, but for VL1 Sol 28 at $1.6 \mathrm{~m}$.

thus obtained from different data, present striking similar features. This gives consistency to the results in the sense that, provided that the meteorological conditions are similar (no baroclinic disturbances and low dust content), the SL TKE budget for any midlatitude northern summertime Sol over flat terrain should not differ notably from the ones presented in Figures 5 and 7.

\subsubsection{VL2 Sol 20}

The synoptic conditions during VL2 Sol 20 were not as steady as for PF Sol 25 and VL1 Sol 28; the atmospheric 

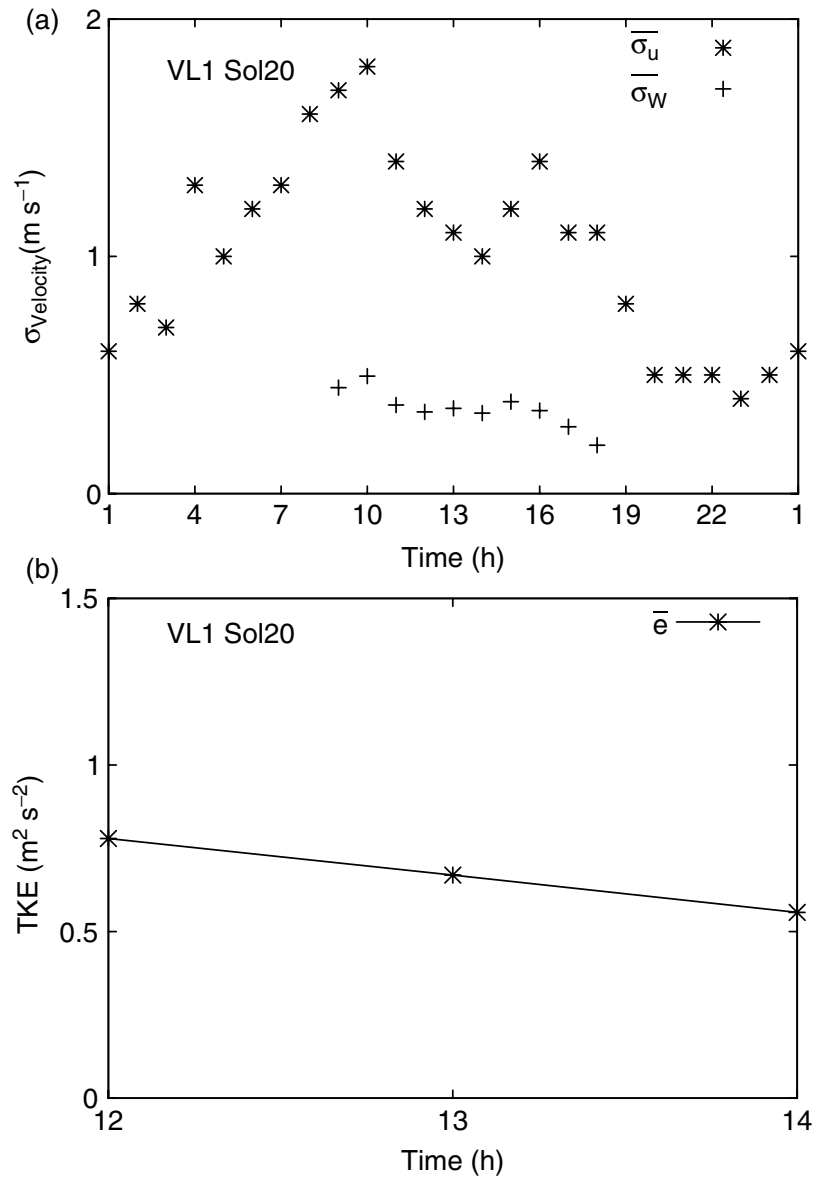

Figure 8. As Figure 4, but for VL2 Sol 20 at $1.6 \mathrm{~m}$.

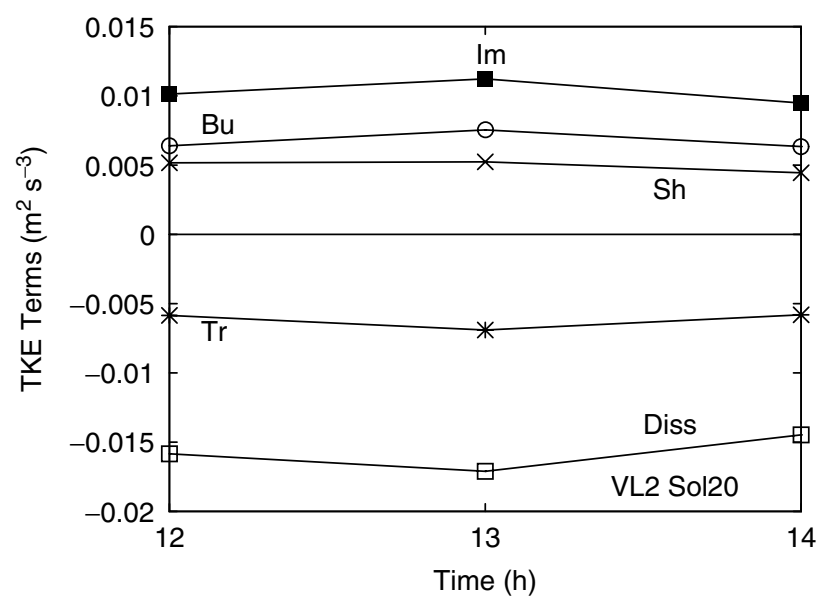

Figure 9. As Figure 5, but for VL2 Sol 20 at $1.6 \mathrm{~m}$.

distortion and also the dust content were higher during the VL2 first summer (Tillman et al., 1994).

These particular atmospheric conditions can be noticed in Figure 8, where the velocity standard deviations and the TKE for VL2 Sol 20 are shown. The behaviour of the horizontal velocity standard deviation differs significantly from that found on PF Sol 25 and VL1 Sol 28. During the central part of the day, both $\overline{\sigma_{u}}$ and the mean horizontal wind $\bar{U}$ (Figure 1) present atypical low values, while those for the early morning and evening are higher. Consequently, TKE presents values below $1 \mathrm{~m}^{2} \mathrm{~s}^{-2}$.
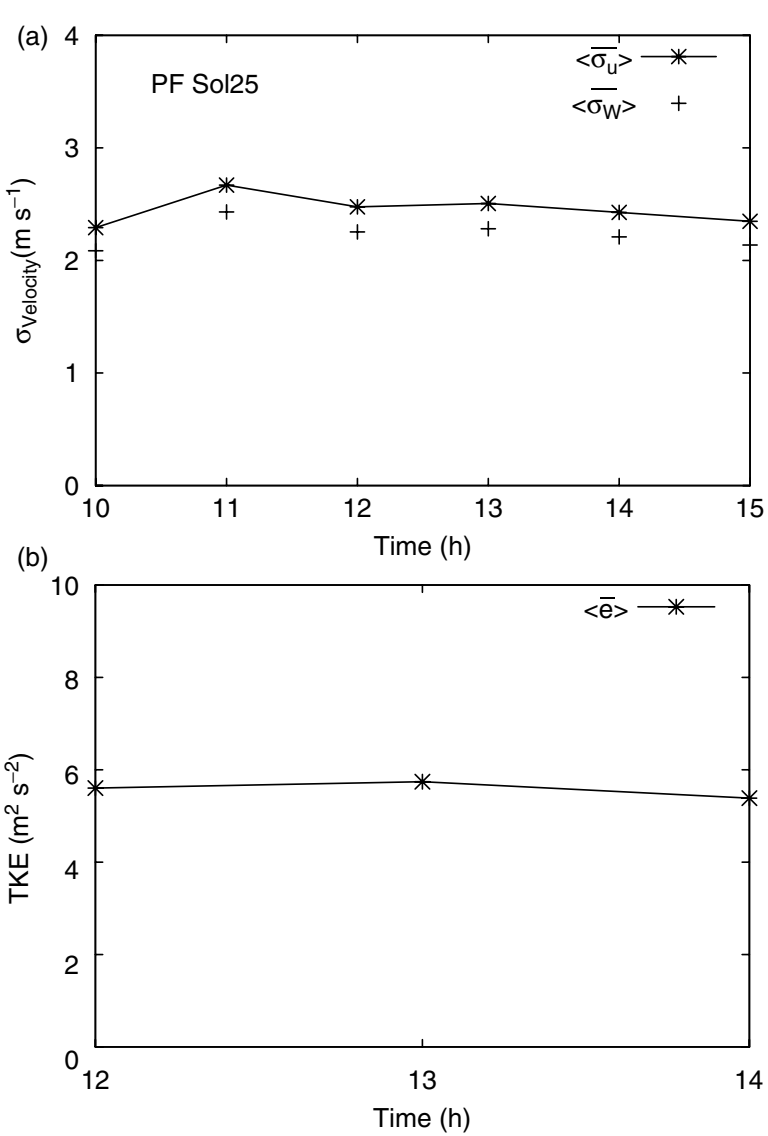

Figure 10. PF Sol 25 convective mixed layer statistics: (a) hourly values of vertically averaged horizontal $\left\langle\overline{\sigma_{u}}\right\rangle$ and vertical $\left\langle\overline{\sigma_{w}}\right\rangle$ velocity standard deviations, and (b) TKE obtained from these at the most convective hours. TKE and horizontal velocity standard deviations are shown as solid lines for clarity, although the estimations are discrete.

Such specific conditions are also noticed in Figure 9, where the SL TKE budget for this Sol is displayed. Shear, with values around $5 \times 10^{-3} \mathrm{~m}^{2} \mathrm{~s}^{-3}$, is no longer the main TKE source, but the imbalance plays that role. Therefore, any of the terms accounting for Im in Eq. (3)-grouped into the imbalance term due to their presupposed non-prominent roles-now plays a leading role. (Section 5 provides further details of Im.) Turning to the other terms involved in the TKE budget, buoyancy is slightly higher than shear, and also contributes to the generation of TKE. The dissipation is still the principal TKE remover, with values $\sim 10^{-2} \mathrm{~m}^{2} \mathrm{~s}^{-3}$, which exceeds by one order of magnitude the shear and buoyancy production. The vertical turbulent transport removes TKE at the same rate that buoyancy generates it, while the storage term, as in the previous Sols, has a negligible impact in the TKE budget.

\subsection{TKE budget in the CML}

The results are similarly arranged as in the SL case: the averaged horizontal $\left\langle\overline{\sigma_{u}}\right\rangle$ and vertical $\left\langle\overline{\sigma_{w}}\right\rangle$ velocity standard deviations are displayed, then the TKE derived from these. Finally, the averaged budget for the TKE is described.

\subsubsection{PF Sol 25}

Figure 10 displays the velocity standard deviations. It is shown that the intensity of the turbulence on the horizontal plane is the same as on the vertical plane, at least when 


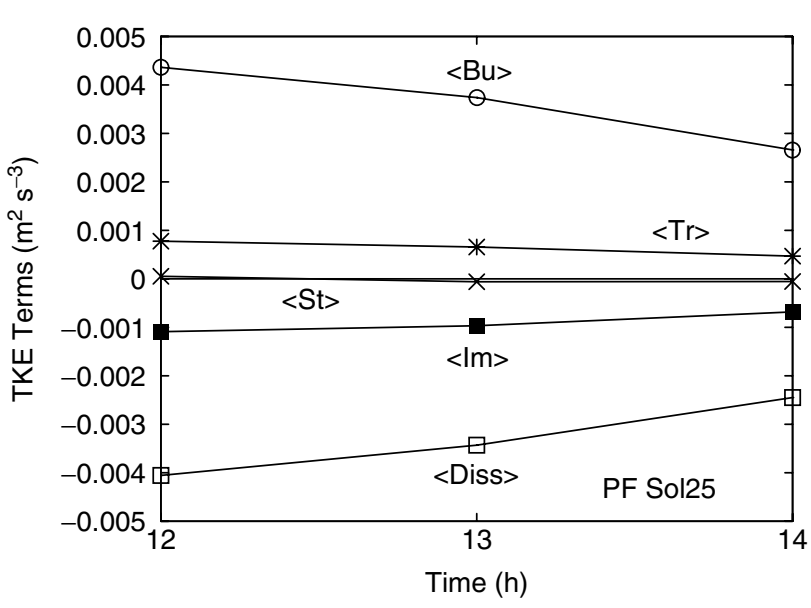

Figure 11. TKE budget in the convective mixed layer for PF Sol 25. The results span the most convective hours (from $1200 \mathrm{~h}$ to $1400 \mathrm{~h}$, local solar time), when the TKE is expected to be totally developed. $\langle B u\rangle,\langle S t\rangle,\langle\operatorname{Tr}\rangle$, $\langle$ Diss $\rangle$, and $\langle$ Im $\rangle$ denote the vertically averaged buoyancy, storage term, vertical turbulent transport, dissipation, and imbalance term, respectively. The shear term $\langle S h\rangle$ has not been included due to its negligible relevance, with values of the order of $10^{-6} \mathrm{~m}^{2} \mathrm{~s}^{-3}$, i.e. one order of magnitude less than the smallest $\langle$ St $\rangle$ term.

comparing averaged values. Both magnitudes lie in the range $2-3 \mathrm{~m} \mathrm{~s}^{-1}$, which closely matches other estimations, such as those obtained by Sorbjan (2007a) for an idealized Martian location, or by Spiga and Forget (2009) for the Gusev Crater. However, our results differ qualitatively from those of Michaels and Rafkin (2002), who found that the turbulence in the vertical was more intense using a LES run for PF conditions. However, all these showed vertical profiles instead of vertically averaged values, which might result in differences from our results.

The averaged TKE, obtained from Eq. (12), is also shown in Figure 10, and can be considered to be in a steady state; its values remain on average close to $6 \mathrm{~m}^{2} \mathrm{~s}^{-2}$. Such averaged values are in accordance with other vertical TKE profiles, such as those shown by Rafkin et al. (2004) obtained using the MRAMS mesoscale model for Beagle 2 landing site, or those derived from a LES run by Tyler et al. (2008) and Spiga and Forget (2009) for the Phoenix and Mars Exploration Rover landing sites, respectively.

We now comment on the CML TKE budget shown in Figure 11. Buoyancy becomes the principal source of TKE (as could be expected due to the vigorous upward thermals existing in the Martian CML), and presents values around $4 \times 10^{-3} \mathrm{~m}^{2} \mathrm{~s}^{-3}$. Dissipation balances buoyancy, this being the main mechanism for removing TKE. The vertical turbulent transport term plays a minor role compared to the dominant mechanisms, with values around $8 \times 10^{-4} \mathrm{~m}^{2}$ $\mathrm{s}^{-3}$. Unlike in the SL, it is positive and therefore, on average, the CML is receiving TKE from the SL, from where TKE is exported upward. The imbalance, which is negative and almost equal in magnitude to the vertical turbulent transport term, represents around $25 \%$ of the buoyancy or dissipation (main mechanisms).

\subsubsection{VL1 Sol 28}

The averaged horizontal and vertical velocity standard deviations, and the corresponding averaged TKE derived from these, are shown in Figure 12. They present values slightly higher than for PF Sol 25, though the qualitative
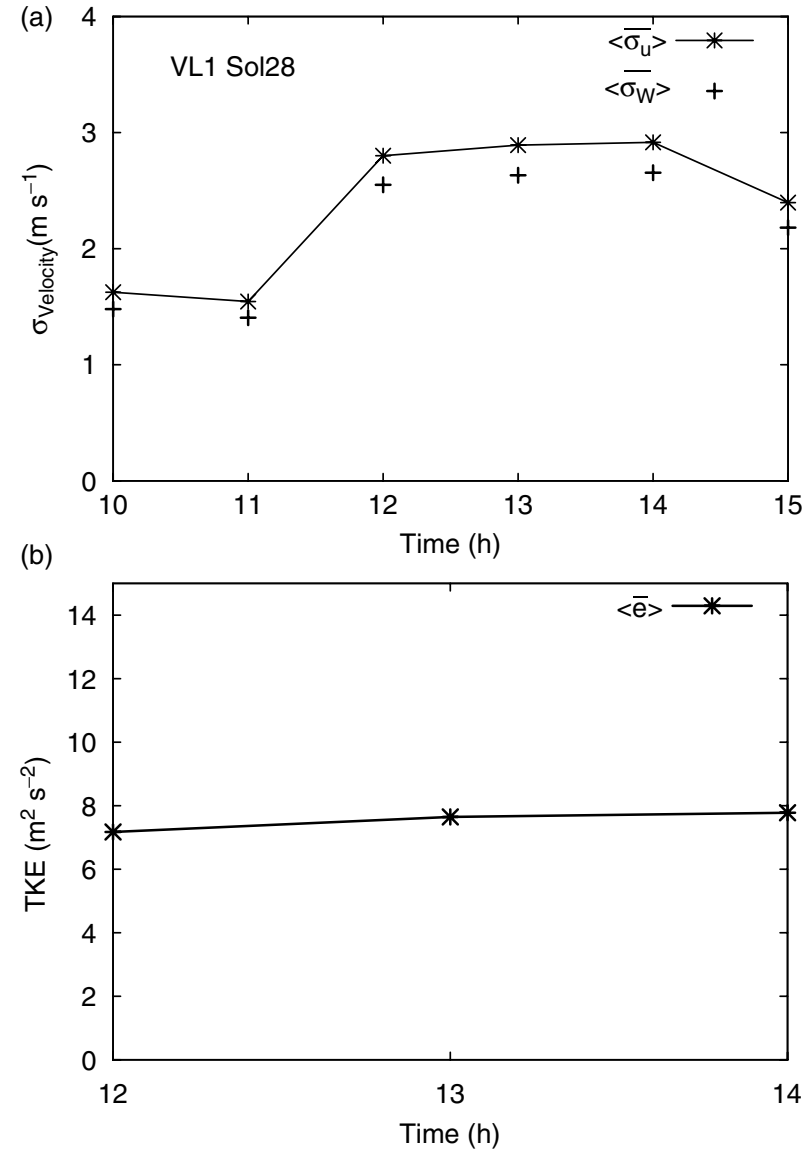

Figure 12. As Figure 10, but for VL1 Sol 28.

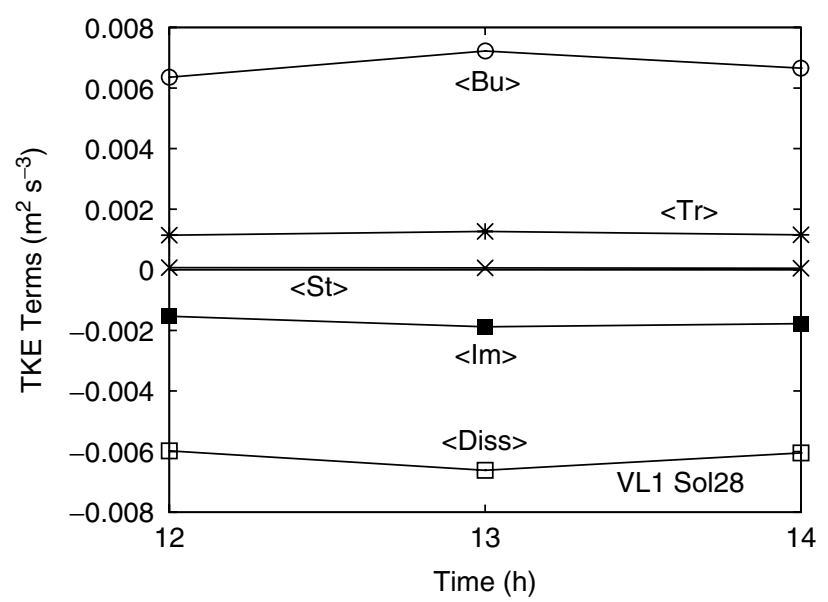

Figure 13. As Figure 11, but for VL1 Sol 28.

behaviour is the same, i.e. turbulence is isotropic (when considering mean values), and TKE can be seen to remain in steady state from $1200 \mathrm{~h}$ to $1400 \mathrm{~h}$, with values around $7 \mathrm{~m}^{2} \mathrm{~s}^{-2}$.

The CML TKE budget for VL1 Sol 28 is shown in Figure 13, and is very similar to that of PF Sol 25, as was also the case for the SL TKE budget. Buoyancy dominates the generation of TKE $\left(\simeq 6 \times 10^{-3} \mathrm{~m}^{2} \mathrm{~s}^{-3}\right)$, and is almost totally balanced by dissipation, the main TKE remover. The vertical turbulent transport is positive, with values $\simeq 1 \times 10^{-3} \mathrm{~m}^{2} \mathrm{~s}^{-3}$, meaning that the CML receives TKE from the SL, though it still plays a minor role. The imbalance term is of the order of the vertical transport term, and about $25 \%$ of the main mechanisms. 

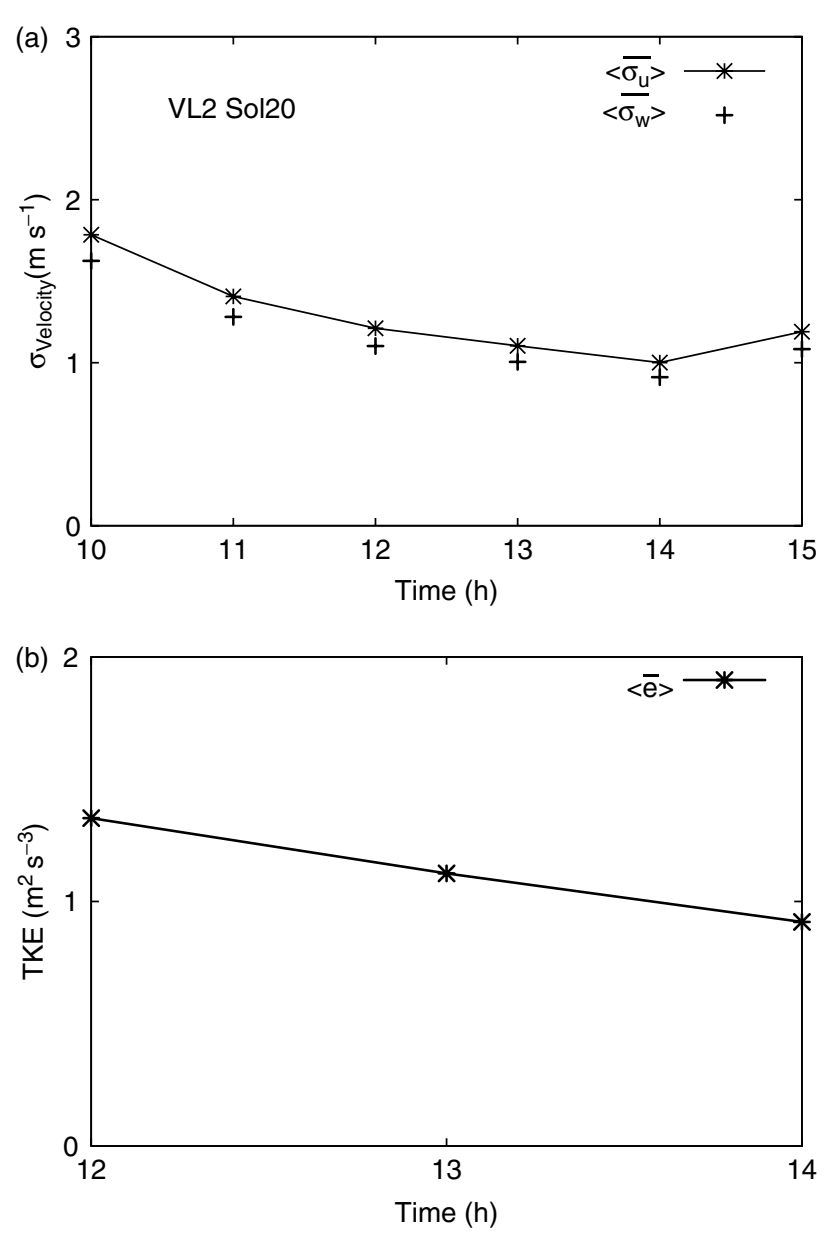

Figure 14. As Figure 10, but for VL2 Sol 20.

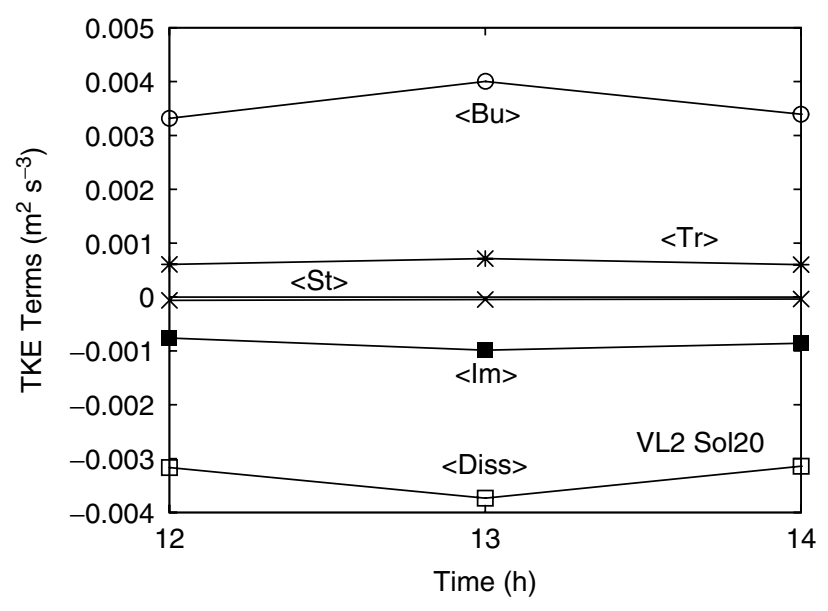

Figure 15. As Figure 11, but for VL2 Sol 20.

Finally, the storage and shear terms are negligible, presenting the same values as in PF Sol 25.

\subsubsection{VL2 Sol 20}

The averaged horizontal and vertical velocity standard deviations, together with the corresponding averaged TKE, are shown in Figure 14. The values are lower than for the VL1 and PF Sols, specially for the TKE, though the isotropy is still maintained. As discussed below, these low values
Table III. Dependencies of the TKE budget terms in the surface layer on (i) the inclusion of a parametrization accounting for the molecular sublayer, (ii) the surface roughness $\left(z_{0} \in(0.1,10) \mathrm{cm}\right)$, and (iii) the ground temperature, $T_{\mathrm{g}}$. The storage term has not been included due its negligible values compared with the rest of the terms.

\begin{tabular}{lccc}
\hline $\begin{array}{c}\text { Molecular } \\
\text { sublayer }\end{array}$ & $\begin{array}{c}\text { Surface } \\
\text { roughness }\end{array}$ & $\begin{array}{c}T_{\mathrm{g}} \\
\text { scenarios }\end{array}$ \\
\hline $\mathrm{Bu}$ & $\searrow$ & $\nearrow$ & $\nearrow$ \\
$\mathrm{Sh}$ & None & $\nearrow \nearrow$ & None \\
$|\mathrm{Tr}|$ & $\searrow$ & $\nearrow$ & $\nearrow$ \\
$\mathrm{Di}$ & None & $\nearrow \nearrow$ & None \\
\hline
\end{tabular}

$\nearrow$ and $\searrow$ denote an increase or decrease of $<30 \%$ of the most likely values shown in Figures 5,7, and 9.

$\nearrow \nearrow$ denotes an increase of $>85 \%$ of the most likely values.

'None' denotes no dependence at all.

are thought to be caused by the particular environmental conditions during this Sol.

We turn to the CML TKE budget for VL2 Sol 20, displayed in Figure 15. Though in this case the imbalance term has not the largest impact on the TKE budget, as was the case in the SL for this Sol, it still represents 30\% of buoyancy or dissipation. Therefore, the weight of $I m$ on the TKE budget in the overall PBL has not a minor role for VL2 Sol 20. We attribute this behaviour to the atmospheric variability and higher amount of dust occurring during the VL2 first summer, which makes the imbalance term across the PBL higher than would be expected under ideal conditions for application of similarity theory. Section 5 will shed light on the origin of the imbalance and its special impact on the TKE budget for VL2 Sol 20.

\subsection{Sensitivity studies for the TKE budget}

Each of the terms obtained for the TKE budget in the last two subsections, both in the SL and CML, have been derived under the most probable scenario in the broadest sense, namely:

(i) a parametrization accounting for the molecular sublayer was added to terrestrial similarity theory,

(ii) the surface roughness was set to a value of $z_{0}=1 \mathrm{~cm}$, and

(iii) the ground temperature corresponded to the most probable scenario shown in Figure 3.

To check the consistency of the results, sensitivity studies regarding the above parameters have been performed. From the data presented in section 2, Monin-Obukhov length $L$, friction velocity $u_{*}$, scale temperature $T_{*}$, convective velocity scale $w_{*}$, and PBL height $z_{\mathrm{i}}$ were obtained in Martinez et al. (2009b), together with their dependence on molecular sublayer parametrization, the surface roughness, and the ground temperature. From those results $\left(L, u_{*}\right.$, $\left.T_{*}, z_{\mathrm{i}}, w_{*}\right)$, and using the described data and methodology, we have obtained values for the TKE budget terms via Eqs (5), (6), (7), (8), (14), (15), (17), and (18). It is now straightforward to study the variation of buoyancy, shear, vertical turbulent transport, and dissipation with respect the surface roughness, the ground temperature, and the inclusion of a parametrization for the molecular sublayer. 
Table IV. As Table III, but for the convective mixed layer. The storage and shear terms have not been included due to their negligible weight in the TKE budget.

\begin{tabular}{cccc}
\hline $\begin{array}{c}\text { Molecular } \\
\text { sublayer }\end{array}$ & $\begin{array}{c}\text { Surface } \\
\text { roughness }\end{array}$ & $\begin{array}{c}T_{\mathrm{g}} \\
\text { scenarios }\end{array}$ \\
\hline $\mathrm{Bu}$ & $\searrow$ & $\nearrow$ & $\nearrow$ \\
$\mathrm{Tr}$ & $\searrow$ & $\nearrow$ & $\nearrow$ \\
$\mathrm{Di}$ & $\searrow$ & $\nearrow$ & $\nearrow$ \\
\hline
\end{tabular}

\subsubsection{Surface layer dependencies}

We show in Table III the dependencies of the TKE budget terms in the SL. A parametrization accounting for the molecular sublayer has been included by assuming a relationship for $z_{0} / z_{0 T}$ (Brutsaert, 1982), with $z_{0 T}$ the surface skin temperature height taking values in the range $10^{-3}-10^{-4} \mathrm{~m}$. The main effect of adding this parametrization has been found to be a surface heat flux decrease. Consequently, the values for the buoyancy and vertical turbulent transport terms become lower, while shear and dissipation remain unchanged. Regarding the surface roughness, it is expected that more horizontal momentum is lost to the ground as the surface becomes more irregular. Thus, shear is observed to grow even one order of magnitude for $z_{0}=10 \mathrm{~cm}$, as does dissipation, since this term is in charge of balancing the production (shear) of TKE in the SL. The effect of the surface roughness on the other terms is less noticeable. Finally, under the highest $T_{\mathrm{g}}$ scenario, the difference from the atmospheric temperature becomes the greatest, and so the convection should be more powerful. Accordingly, buoyancy grows under the highest ground temperature scenario, as does vertical turbulent transport, since the more buoyancy there is to be exported, the better the vertical turbulent transport can perform. Shear and dissipation remain unaltered.

\subsubsection{Convective mixed-layer dependencies}

The dependencies of the TKE budget terms in the CML are shown in Table IV. Buoyancy and turbulent transport decrease with the inclusion of a parametrization for the molecular sublayer, for the same reasons as those accounting for the SL decrease. Dissipation, which balances buoyancy in the CML, also decreases, in order to maintain such balance. On the contrary, all terms increase slightly with the surface roughness. Finally, the warmer the ground is, the stronger the buoyancy becomes, as does the dissipation to balance the buoyancy. In addition, the turbulent transport also increases, since more TKE is being received in the CML via buoyancy in the scenario of highest ground temperature.

\section{Discussion}

The discussion of the results is arranged in two subsections. First, an explanation is presented on the significance of the imbalance, followed by some calculations to clarify which of the component terms are the most likely to account for it. Second, a comparison is given between Earth and Mars regarding the weight of each of the terms involved in the TKE budget.

\subsection{Justification of the imbalance}

Under ideal conditions for the application of similarity theory-horizontal homogeneity, no baroclinic disturbances, and predominance of the turbulent convective heating versus the radiative one-the imbalance term, as defined in Eq. (3), should have a negligible impact on the TKE budget, both in the unstable SL and in the CML.

However, based on our results, the imbalance always plays a non-negligible role. This is because its weight in the TKE budget can be regarded as a measure of how far the topographical and environmental conditions are from the ideal ones. Accordingly, the imbalance plays only a minor role during PF Sol 25 and VL1 Sol 28-both for the SL and the CML-where such ideal conditions were met to a reasonable degree. However, the atmospheric distortion and the dust content during VL2 Sol 20 made the environmental conditions differ notably from the ideal scenario. As a consequence, the imbalance even dominates the budget in the SL, while still plays a non-negligible role in the CML.

We next analyze the most likely terms accounting for the imbalance based on Eq. (3), both in the SL and in the CML, and for the three Sols under study.

\subsubsection{Surface layer}

The SL TKE budget for PF Sol 25 and VL1 Sol 28, shown in Figures 5 and 7, respectively, is basically the same. During both Sols, the imbalance term is close to $5 \times 10^{-2} \mathrm{~m}^{2} \mathrm{~s}^{-3}$, and thus the next justification is applicable to both Sols.

The advective term, supposing $\bar{W} \ll \bar{U}$ and that $\partial \bar{e} / \partial z$ does not vary considerably in the SL (Wyngaard and Côté, 1971), results in $\bar{U} \partial \bar{e} / \partial x$. If we compare this term to the value determined for $\operatorname{Im}\left(\simeq 5 \times 10^{-2} \mathrm{~m}^{2} \mathrm{~s}^{-3}\right)$, it is concluded that a variation of TKE of the order of $1 \mathrm{~m}^{2} \mathrm{~s}^{-2}$ across $100 \mathrm{~m}$ in the horizontal would account for the imbalance, provided $\bar{U}=6 \mathrm{~m} \mathrm{~s}^{-1}$ (Figures 1 and 2). Therefore, though VL1 and PF were moderately flat terrains, such variation of TKE in the horizontal cannot be ruled out, which makes advection likely to account for the imbalance.

The horizontal turbulent transport term is unlikely to account for $I m$. The reason is that the vertical turbulent transport is expected to be more important than the horizontal one, and even the former presents lower values than the imbalance term based on Figures 5 and 7.

Turning to the horizontal shear, we find that it can be simply written as $-\overline{\sigma_{u}^{2}} \partial \bar{U} / \partial x$, where it has been assumed that:

(i) $\overline{\sigma_{u}^{2}}$ is at least one order of magnitude higher than $\overline{\sigma_{w}^{2}}$, $\overline{u^{\prime} w^{\prime}},-\overline{u^{\prime} v^{\prime}}$ and $-\overline{v^{\prime} w^{\prime}}$ (Figures 4 and 6; Table I), and that

(ii) $\partial \bar{U} / \partial x$ should not be lower than $\partial \bar{W} / \partial z, \partial \bar{U} / \partial y$, $\partial \bar{W} / \partial x$, and $\partial \bar{W} / \partial y$.

Thus, a change in $\bar{U}$ around $0.75 \mathrm{~m} \mathrm{~s}^{-1}$ across $100 \mathrm{~m}$, with values of $\overline{\sigma_{u}^{2}}$ based on Figures 4 and 6, would allow the horizontal shear to account for the imbalance term, which cannot be disregarded. Therefore the horizontal shear likely accounts for the imbalance term.

Very little is known about the pressure redistribution term $\bar{\rho}^{-1} \partial \overline{u_{j}^{\prime} p^{\prime}} / \partial x_{j}$, even in the case of the Earth. Based on Wyngaard and Côté (1971), this term may become important in the terrestrial SL TKE budget. Thus, we should 
not rule it out on Mars, since the lower density and the existing turbulent pressure variations might cause this term to account for $I m$. This issue requires a further study which is beyond the scope of this paper.

We proceed to justify the imbalance term of the SL TKE budget for VL2 Sol 20, which presents values around $1.1 \times 10^{-2} \mathrm{~m}^{2} \mathrm{~s}^{-3}$ (Figure 9). Following the same methodology than that used for VL1 Sol 28 and PF Sol 25, and assuming values for $\bar{U}$ and $\overline{\sigma_{u}}$ based on Figures 1 and 8 , respectively, the advective term could account for $I m$, since $\partial \bar{e} / \partial x \simeq 0.5 \times 10^{-2} \mathrm{~m} \mathrm{~s}^{-2}$ would be required for this to happen, a value which may be regarded as attainable. The horizontal turbulent transport term is unlikely to account for it, due to the same reasons as for VL1 Sol 28 and PF Sol 25. On the contrary, the horizontal shear term could justify the imbalance, with $\partial \bar{U} / \partial x \sim 10^{-2} \mathrm{~s}^{-1}$ being necessary, which seems plausible. Finally, the pressure redistribution term cannot be ruled out either.

\subsubsection{Convective mixed layer}

Justifying the imbalance as expressed in Eq. (10) is extremely awkward, since, in order to derive the terms for the CML TKE budget, only surface-based 'inputs' have been used. Nonetheless, some clues can be still obtained, as we next show.

The imbalance for the three Sols under study presents values around $1 \times 10^{-3} \mathrm{~m}^{2} \mathrm{~s}^{-3}$, as can be observed in Figures 11, 13 and 15. For the three Sols, the averaged advective term can be written as

$$
\frac{1}{0.6 z_{\mathrm{i}}} \int_{0.2 z_{\mathrm{i}}}^{0.8 z_{\mathrm{i}}}\left(\bar{U} \frac{\partial \bar{e}}{\partial x}+\bar{W} \frac{\partial \bar{e}}{\partial z}\right) \mathrm{d} z
$$

where just Eq. (9) has been used. Assuming that $\bar{U} \sim 10^{1} \mathrm{~m} \mathrm{~s}^{-1}$ and $\bar{W} \sim 10^{0} \mathrm{~m} \mathrm{~s}^{-1}$, and that their values regarding the order of magnitude can be considered constant with height, we obtain the advective term to be $\simeq 10\langle\partial \bar{e} / \partial x\rangle+1\langle\partial \bar{e} / \partial z\rangle$, where the integral mean value theorem has been used. If the advective term is to account for the imbalance, at least either $\langle\partial \bar{e} / \partial x\rangle \sim 10^{-4} \mathrm{~m} \mathrm{~s}^{-2}$ or $\langle\partial \bar{e} / \partial z\rangle \sim 10^{-5} \mathrm{~m} \mathrm{~s}^{-2}$ is required. From these values, it can be concluded that there are no reasons to rule out the averaged advective term.

The averaged horizontal turbulent transport is not expected to account for $\langle I m\rangle$ instead, since, as was also the case in the SL, the vertical turbulent transport, which is supposed to be higher than the horizontal one, is equal to or smaller than the imbalance, as observed in Figures 11, 13, and 15.

With regards to the horizontal shear, it does not necessarily have to be lower than the vertical shear term-as it had to be in the SL-since the vertical mixing in the CML is expected to be powerful. Thus, although the vertical shear is of the order of $10^{-6} \mathrm{~m}^{-2} \mathrm{~s}^{-3}$, the horizontal shear might account for the imbalance. Finally, we do not dare give explanations for the hypothetical values of the pressure redistribution term because of the lack of sufficient data to study it. Thus, we cannot exclude it accounting for $\langle\operatorname{Im}\rangle$ either.

\subsection{Comparison Mars versus Earth}

Several attempts to characterize the TKE budget have been carried out on Earth, as can be seen in the seminal work of
Wyngaard and Côté (1971) and Champagne et al. (1977) for the SL, and of Lenschow (1974) and Kaimal et al. (1976) for the CML. In all these studies, the TKE budget was studied under fair-weather conditions and over flat terrain, with the objective of deriving generic results-as has also been intended in this work. We next discuss the most remarkable similarities and differences found, under the assumption that the TKE budgets on both planets can be compared, since they have been obtained under similar weather conditions and topography.

Champagne et al. (1977) and Kaimal (1978) studied the TKE budget at $z=4 \mathrm{~m}$, and concluded that shear was the dominant mechanism generating TKE. It was virtually counteracted by dissipation (slightly exceeding shear), which was in charge of leading the removal of TKE. Buoyancy and transport played a minor role, and counteracted each other, the first creating TKE, and the second exporting it upwards. This is the same qualitative behaviour that we have found on Mars close to the ground. However some values were different in those terrestrial studies. They obtained for the buoyancy (expected to be independent of height in the SL) values of the order of $10^{-3} \mathrm{~m}^{2} \mathrm{~s}^{-3}$, which represents one order of magnitude less than our obtained Martian values $\left(\sim 10^{-2} \mathrm{~m}^{2} \mathrm{~s}^{-3}\right)$. This difference lies in the higher Martian surface kinematic turbulent heat flux $\overline{w^{\prime} \theta^{\prime}}$, which is caused by the more vigorous convection existing due to the lower atmospheric density and the higher vertical temperature gradient close to the ground. Shear and dissipation were found to be of the order of $10^{-2} \mathrm{~m}^{2} \mathrm{~s}^{-2}$, which represents one order of magnitude less than our Martian values. However, shear and dissipation mostly decrease as $\sim z^{-1}$, and those terrestrial results were obtained at $4 \mathrm{~m}$, while ours have been at 1.3 and $1.6 \mathrm{~m}$. Thus, the results might be considered still similar.

Lenschow (1974) and Kaimal et al. (1976) studied the TKE budget across the CML from measured discrete values covering the bulk of the CML, and so did Pino and ViláGuerau De Arellano (2008) and Moeng and Sullivan (1994) via LESs. In all cases, given the nature of their research, they could obtain vertical profiles of the TKE budget, and not single averaged values as in this study. Based on all these terrestrial studies, two features have been found to be similar between both planets in the bulk of the PBL under convective conditions: shear production of TKE due to the term $-\overline{u^{\prime} w^{\prime}} \partial \bar{U} / \partial z$ was found negligible, and dissipation was roughly constant and the main TKE remover. Yet, unlike our averaged results, buoyancy and transport were found to be of similar importance, both balancing the dissipation term. In addition, transport was not positive (generation) across the entire CML, but it was negative initially, then becoming positive. If we compare precise values, they all found the dissipation to be of the order of $10^{-3} \mathrm{~m}^{-2} \mathrm{~s}^{-3}$, which matches our results. This is the only term which can be compared easily with our results, since it is expected to remain constant across the CML, and so its discrete values should coincide with the averaged value.

\section{Conclusions}

The Martian TKE budget, both for the unstable SL and the CML, has been determined for the first time from surfacebased data belonging to VL1 Sol 28, VL2 Sol 20, and PF Sol 25. An adaptation of terrestrial similarity theory has been used to address such a budget, which constitutes a new approach to study it. 
The SL TKE budget for VL1 Sol 28 and PF Sol 25 is expected to account for each typical midlatitude northern summertime Sol, when the atmospheric variability and the dust content are usually weak, and thus the environmental conditions are close to the ideal ones for the application of similarity theory. Under such a scenario, we have found shear to be the most important mechanism generating TKE, whereas dissipation is the main mechanism for removing TKE, and barely exceeds the shear production. Both mechanisms are of the order of $10^{-1} \mathrm{~m}^{2} \mathrm{~s}^{-3}$. Buoyancy and turbulent transport, which balance each other, play a minor role, with values one order of magnitude lower than shear or dissipation. Specifically, buoyancy generates TKE (upward surface kinematic heat flux), and turbulent transport removes it by transferring it upwards. The imbalance represents about $30 \%$ of the main mechanisms. It presents positive values to balance the tiny excess of dissipation with respect to shear, and can be likely attributed to the advective term, to the horizontal shear term, and to the unknown pressure redistribution term. Finally, the storage term can be considered negligible with values of the order of $10^{-5} \mathrm{~m}^{2} \mathrm{~s}^{-3}$.

The atmospheric variability and the dust content were higher during the first VL2 summer, which caused the environmental conditions to differ significantly from the ideal ones. Accordingly, the SL TKE budget for the VL2 Sol 20 presents anomalous behaviour, with the imbalance becoming the main mechanism generating TKE, where the advection, the horizontal shear, and the pressure redistribution terms are likely accounting for it.

The CML TKE budgets for the three Sols under study present similar characteristics. Buoyancy plays the major role generating TKE, whereas dissipation virtually balances buoyant production. Both mechanisms are around $5 \times 10^{-3} \mathrm{~m}^{2} \mathrm{~s}^{-3}$. The vertical turbulent transport plays a minor role, presenting values one order of magnitude lower than buoyancy or dissipation. Unlike in the SL, vertical turbulent transport is positive, which implies that, on average, the CML is receiving TKE from the SL, from which it is extracted. The imbalance becomes $25 \%$ of the buoyancy or dissipation (main mechanisms), and can be likely attributed to the advective term, to the horizontal shear, or to the pressure redistribution term. The storage term can be neglected, as can be the shear (vertical) term, with values of the order of $10^{-5}$ and $10^{-6} \mathrm{~m}^{2} \mathrm{~s}^{-3}$, respectively.

In order to accurately address the Martian TKE budget in the SL, simultaneous measurements of temperature, horizontal and vertical wind speed, and pressure at several different heights with a high sampling rate $(>1 \mathrm{~Hz})$ would be required, together with additional measurements of ground temperature and dissipation. Similarly, balloon or aircraft measurements with the previously mentioned characteristics would be needed in the CML as well. Such studies are not affordable yet on Mars, and the current approaches which remain are to operate LESs, or to try to make use of the few in situ measurements taken at the surface. We have developed this last option for the first time, and created a methodology to study the TKE budget from such data.

\section{Acknowledgement}

We are grateful for financial support from the Spanish grant 'Participación Científica en la Misión a Marte Meiga-MetNet Precursor' AYA2009-14212-C05-05/ESP, from a UCM-EEA Abel grant in the call UCM-EEA-ABEL-03-2010, and from
NASA's Exobiology Program. We also thank Dr Feito and Ms de la Rosa for helping in the preparation of the article.

\section{References}

Arya PS. 2001. Introduction to Micrometeorology. Academic Press.

Brutsaert H. 1982. Exchange processes at the Earth-atmosphere interface. In Engineering Meteorology Plate EJ. (ed.) Elsevier: Amsterdam.

Champagne F, Friehe C, LaRue J, Wynagaard J. 1977. Flux measurements, flux estimation techniques, and fine-scale turbulence measurements in the unstable surface layer over land. J. Atmos. Sci. 34: 515-530.

Deardorff JW. 1972. Numerical investigation of neutral and unstable planetary boundary layers. J. Atmos. Sci. 29: 91-115.

Dudhia J. 1993. A non-hydrostatic version of the Penn State-NCAR mesoscale model: Validation tests and simulation of an Atlantic cyclone and cold front. Mon. Weather Rev. 121: 1493-1513.

Hess S, Henry R, Leovy C, Ryan J, Tillman J. 1977. Meteorological results from the surface of Mars: Viking 1 and 2. J. Geophys. Res. 82: 4559-4574.

Hinson DP, Pätzold M, Tellman S, Häusler B, Tyler GL. 2008. The depth of the convective boundary layer on Mars. Icarus 198: 57-66.

Högström U. 1988. Non-dimensional wind and temperature profiles in the atmospheric surface layer: A re-evaluation. Boundary-Layer Meteorol. 42: 55-78.

Kaimal JC. 1978. Horizontal velocity spectra in an unstable surface layer. J. Atmos. Sci. 35: 18-24.

Kaimal JC, Wyngaard J, Haugen D, Coté O, Izumi Y. 1976. Turbulence structure in the convective boundary layer. J. Atmos. Sci. 33: 2152-2161.

Kauhanen J, Siili T, Kauhanen J, Järvenoja S, Savijärvi H. 2008. The Mars limited-area model and simulations of atmospheric circulations for the Phoenix landing area and season of operation. J. Geophys. Res. 113: E00A14, DOI: 10.1029/2007JE003011.

Lenschow D. 1974. Model of the height variation of the turbulence kinetic energy budget in the unstable planetary boundary layer. $J$. Atmos. Sci. 31: 465-474.

Lenschow D, Wyngaard J, Pennell W. 1980. Mean-field and secondmoment budgets in a baroclinic, convective boundary layer. J. Atmos. Sci. 37: 1313-1326.

Määttänen A, Savijärvi H. 2004. Sensitivity tests with a one-dimensional boundary-layer Mars model. Boundary-Layer Meteorol. 113:305-320.

Martínez GM, Valero F, Vázquez L. 2009a. Characterization of the Martian surface layer. J. Atmos. Sci. 66: 187-198.

Martínez GM, Valero F, Vázquez L. 2009b. Characterization of the Martian convective boundary layer. J. Atmos. Sci. 66: 2044-2058.

MEPAG. 2008. 'Summary of the Mars science goals, objectives, investigations, and priorities'. Johnson JR. (ed.) Survey whitepaper, Mars Exploration Program Analysis Group: Pasadena, California. http://mepag.jpl.nasa.gov/reports/index.html

Michaels TI, Rafkin SCR. 2002. Large-eddy simulation of atmospheric convection on Mars. Q. J. R. Meteorol. Soc. 130: 1251-1274.

Moeng CH, Sullivan PP. 1994. A comparison of shear- and buoyancydriven planetary boundary-layer flows. J. Atmos. Sci. 51: 999-1022.

Monin AS, Obukhov AM. 1954. Basic laws of turbulent mixing in the atmosphere near the ground. Trudy Geofiz. Inst. AN SSSR 24: $163-187$.

Pielke RA, Cotton WR, Walko RL, Tremback CJ, Lyons WA, Grasso LD, Nicholls ME, Moran MD, Weslet DA, Lee TJ, Copeland JH. 1992. A comprehensive meteorological modeling system, RAMS. J. Meteorol. Atmos. Phys. 49: 69-91.

Pino D, Vilá-Guerau De Arellano J. 2008. Effects of shear in the convective boundary layer: Analysis of the turbulent kinetic energy budget. Acta Geophys. 56: 167-193.

Rafkin SCR, Haberle R, Michaels TI. 2001. The Mars Regional Atmospheric Modeling System: Model description and selected simulations. Icarus 151: 228-256.

Rafkin SCR, Michaels TI, Haberle RM. 2004. Meteorological predictions for the Beagle 2 mission to Mars. Geophys. Res. Lett. 31: L01703. DOI: 10.1029/2003GL018966.

Richardson MI, Toigo AD, Newman CE. 2007. PlanetWRF: A general purpose, local to global numerical model for planetary atmospheric and climate dynamics. J. Geophys. Res. 112: E09001, DOI: 10.1029/2006JE002825. 
Savijärvi H, Kauhanen J. 2008. Surface and boundary-layer modelling for the Mars Exploration Rover sites. Q. J. R. Meteorol. Soc. 134: 635-641.

Savijärvi H, Määttännen A, Kauhanen J, Harri AM. 2004. Mars Pathfinder: New data and new model simulations. Q. J. R. Meteorol. Soc. 130: 669-683.

Schofield JT, Barnes JR, Crisp D, Haberle RM, Larsen S, Magalhaes JA, Murphy JR, Seiff A, Wilson G. 1997. The Mars Pathfinder atmospheric structure investigation/meteorology (ASI/MET) experiment. Science 278: 1752.

Skamarock WC, Klemp JB, Dudhia J, Gill DO, Barker DM, Wang W, Powers JG. 2005. A description of the Advanced Research WRF Version 2. Tech. Note 468+STR, NCAR: Boulder, USA.

Skyllingstad ED. 2003. Large-eddy simulation of katabatic flows. Boundary-Layer Meteorol. 106: 217-243.

Sorbjan Z. 2007a. Statistics of shallow convection on Mars based on large-eddy simulations. Part 1: shearless conditions. Boundary-Layer Meteorol. 123: 121-142.

Sorbjan Z. 2007b. Statistics of shallow convection on Mars based on large-eddy simulations. Part 2: effects of wind shear. Boundary-Layer Meteorol. 123: 143-157.

Sorbjan Z, Wolff M, Smith MD. 2009. Thermal structure of the atmospheric boundary layer on Mars based on Mini-TES observations. Q. J. R. Meteorol. Soc. 135: 1776-1787.

Spiga A, Forget F. 2009. A new model to simulate the Martian mesoscale and microscale atmospheric circulation: Validation and first results. J. Geophys. Res. (Planets) 114: E02009, DOI: 10.1029/2008JE003242.

Spiga A, Forget F, Lewis S, Hinson D. 2010. Structure and dynamics of the convective boundary layer on Mars as inferred from large-eddy simulations and remote-sensing measurements. Q. J. R. Meteorol. Soc. 136: $414-428$.
Stull R. 1988. An Introduction to Boundary-Layer Meteorology. Springer: Berlin.

Sutton J, Leovy C, Tillman J. 1978. Diurnal variations of the Martian surface layer meteorological parameters during the first 45 sols at two Viking lander sites. J. Atmos. Sci. 35: 2346-2355.

Tillman JE, Landberg L, Larsen SE. 1994. The boundary layer of Mars: Fluxes, stability, turbulent spectra, and growth of the mixed layer. J. Atmos. Sci. 51: 1709-1727.

Toigo AD, Richardson MI, Ewald SP, Gierasch PJ. 2003. Numerical simulation of Martian dust devils. J. Geophys. Res. 108: 5047, DOI: 10.1029/2002JE002002.

Tyler D, Barnes JR, Skyllingstad ED. 2008. Mesoscale and largeeddy simulation model studies of the Martian atmosphere in support of Phoenix. J. Geophys. Res. 113: E00A12, DOI: 10.1029/2007JE 003012.

Undén P, Rontu L, Järvinen H, Lynch P, Calvo J, Cats G, Cuxart J, Eerola K, Fortelius C, Garcia-Moya JA, Jones C, Lenderlink G, McDonald A, McGrath R, Navascues B, Woetman Nielsen N, Odegaard V, Rodriguez E, Rummukainen M, Room R, Sattler K, Hansen Sass B, Savijärvi H, Wichers Schreur B, Sigg R, The H, Tijm A. 2002. 'HIRLAM-5 Scientific Documentation, HIRLAM-5 Project'. Available from: SMHI, S-601767 Norrköping, Sweden.

Van der Hoven I. 1957. Power spectrum of horizontal wind speed in the frequency range from 0.0007 to 900 cycles per hour. J. Atmos. Sci. 14: $160-164$.

Wilson GR, Joshi M. 2000. 'The Martian surface boundary layer'. In Proceedings of 31st Annual Lunar and Planetary Science Conference, 13-17 March 2000, Houston, Texas.

Wyngaard J, Côté O. 1971. The budgets of turbulent kinetic energy and temperature variance in the atmospheric surface layer. J. Atmos. Sci. 28: $190-201$. 